fQA

154
$\mathrm{H} 8$

UC-NRLF

||

\$C 1515143

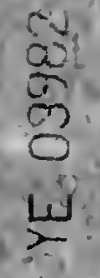




\title{
THE FUNDAMENTAL LAWS OF ADDITION AND MULTIPLICATION IN ELEMENTARY ALGEBRA
}

\author{
Br Enward V. Huntiveton
}

REPRINTED YBOM TRE ANNALS OF MATHKJiTtC, SECOND SERTES, FOL. 8, NO. 1, OCTOBER. 1906

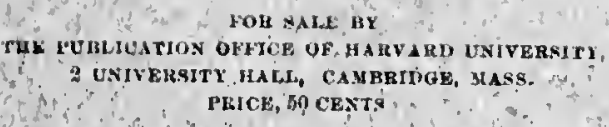





\title{
THE FUNDAMENTAL LAWS OF ADDITION AŃD MULTIPLICA- TION IN ELEMENTARY ALGEBRA*
}

\author{
Br Euwald V. Huntington
}

\section{CONTENTS}

Introduction.

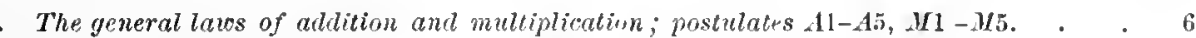

A1. $a+b$ in the system. $M 1 . a \times b$ in the system.

A2. $(a+b)+c=a+(b+c)$. H2. $(a \times b) \times c=a \times(b \times c)$.

A3. (1) If $a+x=a+y$, then $x=y$. M3. (1) If $a x=a y$ and $a+a \neq a$, then $x=y$.

(2) If $x+a=y+a$, then $x=y$. (2) If $x a=y a$ and $a+a \neq a$, then $x=y$.

A4. If $\mu x=\mu y$, then $x=y$.

[A5. $a+b=b+a$.

(See thcorcm 21, page 14.)

§2. Deductions from these laws; theorems 1-20.

IIt. (1) $a(b+c)=a b+a c$

(2) $(b+c) a=b a+c a$.

M5. $a b=b a$.

Thc zcro-element. The unit-clement. Oppositc clements; snbtraction. Reciprocal elcments; division. The so-called inaginary mits.

\$3. Further deductions: use of numerical operators; theorens 21-50.

Multiples of an clement; use of the intcgral numbers as coeflicients. Submultiples and rational fractions of an element; use of the rational numbers as coeflicients. Powers of an elcment; nsc of the intcyral numbers as cxponents. Ejuations of the $\mu$ th degrec in $x$.- Redundancy of the commutative law for addition.

\$4. Special laws of addition and multiplication; postutates $E 1-E 6, F$. . . .

$E$ 1. Existencc of a unit-elcment

$E^{2}$. Existence of a zero-elemcnt.

$E 3$. Fxistence of the oppositc of the unit-element.

$E 4$. Existcnce of the submultiplcs of the nnit-clement.

$E$ 5. Existence of the imaginary units.

$E$ 6. Existencc of a root of any algcloraic equation with integral coefficicnts.

$F$. Non-existence of any other clements besides those requircd by the "cxistcuce-postnlatcs" and postulates $A 1, M 1$.

Particular types of elementary algebra.

The algebra of positive integers $(E 1 ; E)$; of positive integcrs with zero $(E 1,2 ; F)$; of all integers $(E 1,2,3 ; F)$. The algebra of positive rationals $(E 1,4 ; F)$; of positive rationals with \%cro $(E 1,2,4 ; F)$; of all rationals $(E 1,2,3,4 ; F)$. The algebra of complex quantities with integral cocficicnts $(E 1,2,3,5 ; F)$; with rational coefticients $(E 1,2,3,4,5 ; F)$. 'The algebra of all algebraic quantities $(E 1,2,[3,4,5] 6 ; F$,$) .$

\$5. Examples of systems which satisfy some but mu all of the general lairs of $\$ 1$; proofs of independence.

\$6. Proofs of theorems in $\$ \$ 2-4$.

* Thls articlc contains part of a paper prescnted by the writer to the Americau Mathematical Society on Dec. 28, 1905. 


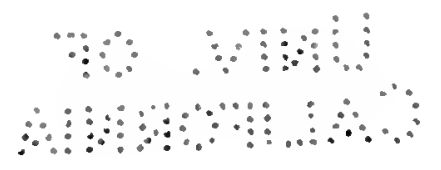

\section{INTRODUCTION.}

Elementary algebra may be regarded, for the purpose of the present paper, as a body of propositions concerning the sums and products of numbers; * these propositions are not independent, but can all be deduced from a few fundunental propositions, or axioms, which are accepted as self-evident properties of number, just as the propositions of geometry can all be deduced from a few fundamental propositions, or axioms, which are accepted as selfevident properties of space.

The primary ohject of this paper is to present a list of fundamental propositions for algebra, from which, on the one hand, all the other propositions of algebra cau be deduced, and in which, on the other hand, no superfluous items are included, - a list, in short, which is sufficient, and free from redundrmcies.

The first propositions which suggest themselves for this purpose are the ten "general laws" numbered $41-1 \overline{1}, M 1-M 5$, in $\$ 1$; all these laws will be recognized as familiar and obviously true propositions concerning numbers.

The next step is to see what propositions follow from these laws by logical deduction. But here the question at once arises: How can we be sure that our deduction is riggorous: How can we he sure that we do not employ, in our reasoning, some other properties of numbers besides those expressly stated in the axions? The only way to avoid this danger is to think of our fundamental laws, not as axiomatic propositions about numbers, but as blank forms in which the letters $", b, c$, ctc. may denote any objects we please and the symbols + and $x$ any rules of combination ; $\dagger$ such a blank form will become a proposition only when a definite interpretation is given to the letters and symbols-indeed a true proposition for some interpretations and a false proposition for others. (Thus, the blank form " $"+b=b+u$ "

* It will greatly assist the reader if he will, from the start, think of multiplication, not as repeated adaition. but as a separate operation, having no connection with addition except through the distributive law, $a(b+c)=a b+a c$.

Many propositions of alyebra concern also the relation of order (or" of "greater" and less") between two numbers; these propositions are not considered however in the present paper, which stops at the point where the intruduction of the relation of order seems necessary. third.

$+d$ rule of eombination is any rule or convention by whieh two objects determine a 
is a true proposition if $a$ and $b$ signify numbers, and + the ordinary addition of numbers; but it is a false proposition if " and $b$ signify rotations of a plane about various axes perpendicular to it, and t the suceession of two sucl rotations.) The deduetions made from sueh blank forms must necessarily be purely formal, and hence will not be affeeted by the troublesome connotations whieh would be sure to attreh themselves to any conerete interpretation of the symbols.

Fron this point of view our work becomes, in reality, mueh more general than a study of the system of numbers; it is a study of any system which salisfies the conditions lrid down in the generm luess of \$1.* As a matter of faet, there are many such systems, all of which are usually ineluded under the general name of algcbra. Thus, there are the various different systems of numbers - the positive integral numbers, the rational numbers, the complex numbers, ete., - all of which, when + and $x$ are detined in the ordinary way, satisfy all these laws. Then there is the system of points (or vectors) in a plane, witl their "sunı" and "produets" defined as in Argand's diagram (see the end of this introduction). Another striking example is the system of all rational numbers, with the "sum" of " and $b$ defined as $a+b+1$ and their "product" as $(d)+(l e+b)$; $\dagger$ a brief computation will show that this strange system also satisfies all the laws of $\$ 1$.

Every system which has the properties stated in the fundanental laws will have also all the properties formally deduced from those laws. The system of natural numbers, with ordinary addition and nultiplieation, appears, therefore, as merely a special case of the general class of systems whose properties are liere studied.

The objeet of the paper may now be more precisely stated in the following form: Given a cless of elements with two rules of combination, what conditions must such a system sut isfy in order to be formully equirulent to one of the systems of ordinur!y relgehre? The first eonditions whieh we impose are naturally the ten "general laws" numbered $A 1-A_{5}$, $M(M-M 5$, in $\$ 1$; but these laws are to be regarded no longer as "axioms," since they are merely blank forms, not in themselves either true or false, but ratler as "postulates," because we

* By a "system," il this connection, we mean any class of entities anong which two rules of combination are established. - The entities which belong to the class are called the "elements" of the elass, or of the system.

†Trans. Amer. Math. Soc., vol. 6 (1905), p. 225. 
"demand," arbitrarily, that the system considered shall eonform to these conditions."

It appears at once, howerer, that therc are many different types of elcmentary algebra (for example the algebra of the positive integers, the algebra of the rational numbers, the algebrit of veetors, ete.), and that the ten general laws of $\$ 1$ are not sufficient to determine any particular type. We therefore. add, in $\$ 4$, a list of speeial laws (postulates $E 1-E 6$ ), which serve to distinguish the various types from one another. This $\$ 4$ thus eompletes the main object of the paper. Special attention may be ealled to the diseussion of the notion of isomorphism between two systems, and the notion of a sufticient, or categorical, set of postulates for a partieular type of algebra (see page 26), whieh are of fundamental importanee in this eomuection.

Finally, in $\$ 5$, the independence of the general laws is established, so that we may be sure that the list contains no ledundancies. The method for establishing the independenee of a set of postulates consists in cxhibiting, in the case of each postulate, an example of a system which satisfies all the other postulates of the set, but not the one in qucstion. $\dagger$ These systems may be ealled pseudo-algebras, since they fail to be true algebras in respect to some single item in the speeifications.

Ineidentally, the paper contains a rigorous development of the rational number-systen, starting from the sequenee of the natural numbers. The various kinds of numbers are introduced primarily as operators, to indicatc repeated addition, repeated multiplication, etc., performed on the elements of the original systent; but rules of eombination are defined anong thesc operators in such a way that they become themselves examples of systems which satisfy all the laws of $\$ 1$. Moreover, all the examples used in $\$ 5$ are eonstructed out of material offered by thesc number-systems, so that no part of the paper (except the introduction, and the proof of the final paragraph in $\$ 4$ ) presupposes any knowledge of mathematies whatever, beyond the ability to recite the familiar sequenee of natural numbers: $1,2,3$, etc.

* Any set of consistent postulates might be used as the basis of an abstract deductive theory; but only those sets of postulates are worth studying which are capable of some interesting concrete interpretation. If preferred, the postnlates may be called "assumptions," or "hypotheses;" cf Trins. Amer. Hath. Sor., vol. 5 (190t), p. 283.

†This method las become famillar in the last ten years through the works of Peano, Pieri, Padoa, Hilbert, and others.

$\ddagger$ It is customary, however, to extend the word algebra so as to include any system which satisfles postulates $A l, 2,3,4.5 ; M 1,4$; and $E 1$. 
The signs " $="$ and " $\neq$ " are used to denote equality and inequality, respectively; two elements are said to be cqual when eitler can replace the other in every proposition in which it occurs.

It is needless to add that the paper eontains no new theorems in so old a subjeet as elcmentary algebra; the only part of the paper which has any claim to originality is $\$ 5$, containing the proofs of independence.

For bibliographical references, the readcr is referred to the Transactions of the American Mathematical Society, vol. 3 (1902), p. 264 ; vol. 5 (1904), p. 288 ; vol. 6 (1905), p. 209 ; to H. Hankel's Theorie der complexen Zahlensysteme (1867) ; to Stolz and Gmeiner's Theoretische Aritlemetik (1902); and to artieles in the Encyclopüdie der mathematischen Wissenschaften.

\section{Illustrative example.}

In order to have before the reader a coneretc example of a system which satisfies all the postulates, we cite at once the familiar geometric examplc of the ordinary eomplex quantities, or veetors in the plane (Argand's diagram). In this system the elass of elements considered is the class of all the points in the plane, ineluding a speeial point $O$, called the origin, and another spccial point $U$, whose distance from $O$ is called the unit-distance.

The point $A+B$ is defined as the point arrived at by starting from $A$ and taking a step equal in length and direetion to the step from $O$ to $B$.

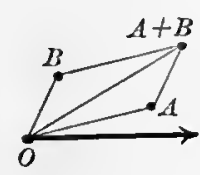

FIG. 1.

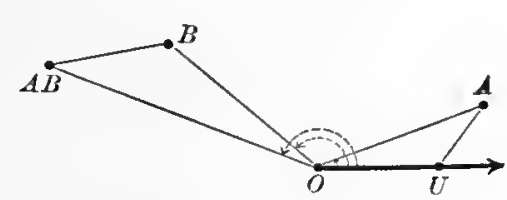

FIG. 2.

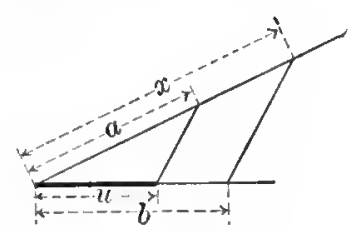

rli, 3.

The point $A \times B$ is defined as the point whose "angle" (from $O U$ ) is the sum of the "angles" of $A$ and $B$ and whose "distance" (from $O$ ) is the product of the "distanees" of $A$ and $B$. Here if $a$ and $b$ arc the distances of $A$ and $B$ respectively, then the "produet," $x$, of $a$ and $b$, is to be constructed geometrieally from the proportion $x: a=b: a$, , where $u$ is the unit-distance (see figure 3 ).

With an elcmentary knowledge of plane geometry, including the properties of sinilar triangles, onc can readily show that this system satisfies all the postulates $A 1-A 5, M 1-M 5$, in $\$ 1$, and also the "existenee-postulates" $E 1-E 5$, in $\$ 4$; the proof for $E 6$, however, is more difficult. 
[The "product" of two distmees, $a$ and $b$, with respect to the unit-distance 1 , may also be defined as follows: In the special case in which $a$ and $b$ are commensurable with $u$, say $a=(\kappa / \mu) u$ and $l=(\lambda / \nu) u$, their product is defined as $(\kappa \lambda / \mu \nu) u$. In any case, there are sequences of commensurable distances which approach a and $b$ as limits:

$$
a=\lim \left[\lambda_{1} \frac{u}{2}, \lambda_{2} \frac{u}{2^{2}}, \lambda_{3} \frac{u}{2^{3}}, \ldots\right], b=\lim \left[\mu_{1} \frac{u}{2}, \mu_{2} \frac{u}{2^{2}}, \mu_{3} \frac{u}{2^{3}}, \ldots\right] ;
$$

then the product of $a$ and $b$ is defined as the linit of the seqnence

$$
\lambda_{1} \mu_{1} \frac{u}{2^{2}}, \quad \lambda_{2} \mu_{2} \frac{u}{2^{4}}, \quad \lambda_{3 \mu^{3}} \mu_{3} \frac{u}{22^{6}}, \ldots
$$

If this definition appear's less simple than the geometrie definition given above, it nlay be remembered that the properties of similar triangles, on which the veometric contruction depends, are usually established by the considera- tion of limits of infinite sequences of precisely this charater.]

\section{\$1. THE GENERAL LAWS OF ADPITION ANI MULTHPICATION.}

Wo consider a clusis of elements, denoted by $a, b, c$, ete., and two rules of combination, called addition $(+)$ and multiplication $(X)$; and npon this system we impose the following conditions, expressed in the postulates numbered I, $A 1-A 5, M 1-1 / 5$.

The consistency of these postulates is shown by the examples given in the introdnction and in $\$ 3$; their independence will be established by the examples given in $\$ 5$.

Any system which satisfies these postulates $A 1-A 5, M 1-M 5$ is said to abey the yeneral laws of elementury algebra as regards addition and multiplication. Various special types of systems of this kind will be distinguished by means of further postulates in $\$ 4$.

In order to exclnde the obviously trivial cases of an empty class and a class containing only a single element, we adopt, first of all,

Postulate I. The class contains at least two elements.

This postulate will be assumed without further mention throughout the paper. 
The laws of addition.

Postulate A1. If $a$ and $b$ are elements of the class $(a=b$ or $a \neq b)$, then $a+b$ is likewise an element of the class, uniquely determined by a and $b$ in their given order, and called the "sum, a plus b."

The operation of finding $a+b$ when $a$ and $b$ are given is called "addition ;" the clements $a$ and $b$ are called the "terms" of the sum $a+b$.

Any system whieh satisfies this postulate $A 1$ may be called a closed system with respect to addition, since the sueeessive addition of any number of elements does not take us outside the system. When sums of three or more elements are eonsidered, parentleses are employed with obvious signifieance, as in $[(a+b)+c]+d$, etc. It should be notieed, however, that as far as postulate $A 1$ is eoncerned, $a+b$ is not neeessarily the same element as $b+a$ (see postulate $A 5$ ).

Postulate A2. Thromghout the system,

$$
(a+b)+c=a+(b+c) \text {. }
$$

This is the associative law for addition.* In view of this law, parentheses may be removed or inserted at pleasure in a sum of any number of terms.

$$
\begin{aligned}
& \text { Postulate A3. (1) If } a+x=a+y \text {, then } x=y . \\
& \text { (2) If } x+a=y+a \text {, then } x=y .
\end{aligned}
$$

These may be ealled the laws of cancelation for addition. $\dagger$ Either (1) or (2) is deducible from the other by the aid of the eommutative law for addition (see postulate $A 5$ ), but so many theorems ean be proved from $A 1,2,3$ without the aid of that law that it has seemed worth while to state both parts of $A 3$ in this manner.

Postulate A4. If $\mu x=\mu y$, where $\mu$ is any posilive integer, then $x=y$.

This postulate will be used first in eonnection with theorem 26 ; the notation, which indieates repeated addition, will be explained in theorem 21 . The postulate may be called the law of non-circularity, since, as we shall see

* The words "associative," "commutative," and "distributive" have been in general use sinee the middle of the nineteenth eentury. See II. Hankel, Theorie der complexen Zahlensysteme (I867), p. 3, fuot-note.

† Cf. Trans. Amer. Wath. Soc., vol. 6 (1905), p. 212. 
in theorem 26 , it prevents a repeated summation from returning, so to speak, into itself. (For a "weaker" postulate, whieh can be used, under eertain eonditions, in place of postulate $A 4$, see appendix 1.)

[Postulate A5. Throughont the system,

$$
a+b=b+a \cdot]
$$

This is the commutrtive lav for addition." "This postulate is placed in brakets, beeause it will prove to be deducible from $A 1,2,3$, with the aid of some of the laws of multiplieation, and is therefore redundant when the list of postulates is taken as a whole (see page 25.)

\section{The laves of multiplication.}

Postulate M1. If a and $b$ are elements of the class $(a=b$ or $u \neq b)$, then $a \times b$ (written also $a \cdot b$ or simply $a b$ ) is likevise an element of the class, uniquely determined by $a$ and $b$ in their given order, and called the "prouluet, a times $b . "$

The operation of finding $a \times b$ when $a$ and $b$ are given is ealled "multiplieation;" the elements $a$ and $b$ are ealled the "fuetors" of the product $a b$. Parentheses aro used as in addition.

Postulate M2. Throughout the system,

$$
(a \times b) \times c=a \times(b \times c) .
$$

This is the associative law for multiplication. In view of this law, parentheses may be removed or inserted at pleasure in a produet of any number of faetors.

$$
\begin{aligned}
& \text { Postulate M3. (1) If } a x=a y \text { and } a+a \neq a \text {, then } x=y \text {. } \\
& \text { (2) If } x a=y a \text { and } a+a \neq a \text {, then } x=y \text {. }
\end{aligned}
$$

These are the laws of cancelation for multiplication. Either (1) or (2) is dedueible from the other by the aid of the eommutative law for multiplication (postulate $M 5$ ); both parts are included, however, for the sake of the deduetions which can be unade from $M 1,2,3$ without the aid of $M 5$. The restrietion " $u+a \neq u$ " may be written " $a \neq \mathbb{I I} "$ after the definition of the zeroelement is obtained, in theorem 1. (For a "weaker" postulate which ean be used, under eertain eónditions, in place of postulate $M / 3$, see appendix 1.)

* See footnote* on preccding page. 
Postulate, M4. Throughout the class,

$$
\begin{aligned}
& \text { (1) } a(b+c)=a b+a c, \text { and } \\
& \text { (2) }(b+c) a=b a+c a .
\end{aligned}
$$

These are called the distributive laus " for multiplication with respect to addition. Either (1) or (2) is deducible from the other by the aid of M5; both parts are included, hoverer, for the same reason as in the case of postulate $M 3$.

Postulate M5. Throughout the system,

$$
a \times b=b \times a .
$$

This is the commutative law for multiplication. Unlike the commutative law for addition, this postulate is independent of all the preceding postulates.

These ten postulates, $A 1-A 5, M 1-M 5$, are the general laws of addition and multiplication in elementary algebra. The immediate consequences of thesc laws are developed in the next section.

\section{§2, DEDUCTIONS FRON THESE LAWS.}

Sections 2-3 contain the most important of the deductions which can be drawn from the postulates $A 1-A 5, M 1-M 5$. The precise postulates on which the proof of each theorem depends are stated in brackets after the number of the theorem; to avoid interruption in reading the paper, the proofs themselves, whenever needed, are collected in $\$ 6$ below.

\section{The zero-element.}

T'eorem 1, and Definition. $[A 1,2,3$.$] It follows from postulates$ $A 1,2,3$ that there cannot be more than one element $z$ such that $z+z=z$; if there is any sucl element, it is called the zero-element of the system, and denoted by $\mathbb{1}$; that is,

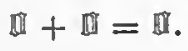

(Proof on p. 35 ; on the use of the symbols $\mathbb{1}$ and 0 , see $\S 3$, below.)

This definition of the zero-element, which is suggested by Benjamin Peirce's definition of an "idempotent" elenent, $\dagger$ is somewhat simpler than the more usual definition, which is based on the property liere stated as theorem 2.

* See footmote * on page $i$

† B. V'eirce, Linear Associative Algebra, 1874. 
Theorem 2. $[A 1,2,3$.$] If there is a rero-element, \mathbb{1}$, then

$$
u+\mathbb{U}=\| \text { and } \mathbb{U}+u=a
$$

for every element $u$; and eonversely, if $u+x=u$ or $x+u=u$, then $x=\mathbb{I}$. (Ploof on p. 36.)

On aeeount of this additive property, the zero-element is often ealled the "modulus" of addition."

Theorem 3. $[A 1,2,3 ; M 1,4$.$] If there is a zero-element, 0$, then

$$
a \times \mathbb{U}=\mathbb{U} \text { and } \mathbb{I I I} \times \cdot \|=\mathbb{I I I}
$$

for every element $a$. (Proof on p. 36.)

This theorem expresses the multiplicative property of the zero-element.

Theorem 4. $\left[A 1,2, ., ; M 1,3_{1}\right.$ or $\left.3_{2}, 4.\right]$ If $a b=\mathbb{0}$ then either $a=\mathbb{I}$ or $b=\mathbb{a}$. In other words, a produet $a b$ eannot be the zero-element, unless at least one of its factors, " or $b$, is the zero-element. (Proof on p. 36.)

This theorem is of considerable importanee, and may be ealled the law of the zer" product (compare appendix 1).

The unit-clement.

- Theorem 5, and Definition. [M1, 2, 3.] It follows from postulates $M 1,2,3$ that there cannot be more than one element $u$, different from the zoro-element, and such that $u \times u=u$; if there is any such element, it is ealled the unit-element of the system, and denoted by 1 ; that is,

$$
\mathbb{b} \times \mathbb{1}=\mathbb{} \quad(\mathbb{1} \neq \mathbb{I}) \text {. }
$$

(Proof on p. 36 ; on the use of the symbols $\downarrow$ and 1 , see $\$ 3$, below.)

Corollary. If $a \times a=\|$ and $\| \neq \mathbb{1}$, then $"=\mathbb{1}$.

This definition of the unit-element is due to B. Peiree (loe. cit.) ; the more usual detinition is here given as theorem 6 .

Therem 6. $[M 1,2,3$.$] If there is a unit-element, \mathbb{1}$, then

$$
" \times \&=" \text { and } \& \times u="
$$

for every element $"$. (Proof on p. 36.)

On aceount of this property, the unit-element is often called the "modulus" of multiplieation. p. 54.

* Cf. H. Mankel, loc. cit., p. 23; also Stolz and Gmeiner, Theoretische Arithmetik (1902), 
Theorem 7. $[A 1,2,3 ; M 1,2,3,4$.$] \quad Conversely, if a x=a$ or $x a=a$, and $a \neq \mathbb{a}$, then $x=\mathbb{1}$. (Proof on p. 36.)

\section{Opposite elements. subtraction.}

Lemma. $\quad[A 1,2,3$.$] \quad It follows from postulates A 1,2,3$ that if $a+b=\mathbb{a}$, then $b+a=\mathbb{a}$; henee we may speak of two elements as having a zero sum, without ambiguity in regard to the order of the terms, even before assuming the commutative law $A 5$. (Proof on p. 36.)

Theorem 8, and Definition. $[A 1,2,3$.$] Given any element a$, there eannot be more than one element $x$ such that the sum of $x$ and $a$ is $\mathbb{1 0}$; if there is any such element, it is ealled the opposite of $a$, and denoted by $-a$, or simply by $-a$; that is,

$$
a+(-a)=(-a)+a=\mathbb{l} \text {. }
$$

Any two elements whose sum is the zero-element are ealled a pair of opposite elements.

Corollary. If there is a zero-element, then $-\mathbb{I}=\mathbb{1}$; and if $a$ is an element whieh has an opposite, then $-(-a)=a$.

The opposite of an element $"$ is eommonly ealled the "negative" of $a$; it seems preferable, however, to reserve the term negative for use in the phrase "positive and negative elements." "

Coneerning the multiplieation of opposites we have:

Theorem $9 . \quad[A 1,2,3 ; M 1,4$.$] If a$ and $b$ are elements whieh have opposites, then

$$
(-a) \times b=a \times(-b)=-a b \text {, and }(-a) \times(-b)=a b \text {. }
$$

(Proof on p. 36.)

The following theorems are the first whieh require the eommutative law for addition (postulate $A 5)$ :

Theorem 10. $[A 1,2,3,5$.$] If a$ and $b$ are elements whieh have opposites, then

(Proof oll p. 36.)

$$
\longleftarrow(-a)+(-b)=-(a+b) \text {. }
$$

* The distiuction between positive and négutive clements involves the notion of order, and will therefore not he discussed in the present paper. (An clement $a$ is called positive or negative aecording as $a+a>a$ or $a+a<a_{*}$ ) It should be mentioned, however, that in many cases the relation of order is deflnable in terms of addition, or of addition and multiplication; see especially O. Veblen, Trans. Amer. Math. Soc., vol. 7 (1906), pp. 197-199. 
Theorem 11, and Definition. [A1,3,5.] Given any elements $a$ and $b$, there cannot be more than one element $x$ sueh that $a=b+x=x+b$; if there is any sueh element it is ealled the remainder, a minus $b$, and denoted by $a-b$; that is,

$$
a=b+(a-b)=(a-b)+b .
$$

The operation of finding $a-b$ when $a$ and $b$ are given is ealled "subtraetion :" the definition of $-a$ in theorem 8 is a speeial ease.

Theorem 12. $[A 1,2,3,5$.$] If the remainders in question exist,$ then $a+(b-c)=(a+b)-c ; a-(b+c)=(a-b)-c$; and $a-(b-c)$ $=(a-b)+c$. Moreover, if there is a zero-element, then $a-\mathbb{I I}=a$ and $a-\imath=\mathbb{I} ;$ and if $-x$ exists, then

$$
u+(-x)=a-x \text { and } a-(-x)=a+x .
$$

(Proof on p. 3i.)

Theorem 13. $[A 1,3,5: M 1,4$.$] If b-c$ exists, then

$$
a(b-c)=a b-a c \text { and }(b-c) a=b a-c a \text {. }
$$

(Proof on p. 37.)

\section{Reciprocal elements. Division.}

Lemma. $[M 1,2,3$.$] \quad It follows from postulates M 1,2,3$ that if $a b=1$, then $b a=\mathbb{1}$; henee we may speak of two elements as having a unit product, without ambiguity in regard to the order of the faetors, even before assuming the eommutative law 145. (Proof on p. 37.)

Theorem 14, and Definition. [1/1,2,3.] Given any element $\alpha$, there eannot be more than one element $y$ such that the prodnet of $y$ and $a$ is $\mathbb{1}$; if there is any sueh element, it is ealled the reciprocal of $a$, and denoted by $\frac{\mathbb{l}}{a}$, or $1 / a$; so that

$$
a\left(\frac{\mathfrak{l}}{\prime \prime}\right)=\left(\frac{\mathbb{d}}{a}\right) "=\mathbb{1}
$$

(Proof on p. 37.) Any two elements whose produet is the unit-element are called a pair of reeiproeal elements.

Remark. In view of theorem 3 , it is evident that if $a=\mathbb{1}$, then no recip)rocal of $a$ exists in any system that satisfies postulate $M 4$. 
Theorem 15. $[A 1,2,3 ; M 1,2,3,4$.$] If a$ is an element which has an opposite and a reciprocal, then

$$
\frac{1}{-a}=-\frac{1}{a}
$$

(Proof on p. 37.)

The following theorems are the first which require the commutative law for multiplication (postulate $M 55$ ) :

Theorem 16. $[M 1,2,3,5$.$] If a$ and $b$ are elements which have reciprocals, then

(Proof on p. 37.)

$$
\frac{1}{a} \times \frac{1}{b}=\frac{\mathbb{1}}{a b}
$$

Theorem 17, and Definition. $[M 1,3,5$.$] Given any elements a$ and $b$, $b$ not zero, then there eannot be more than one element $y$ such that $a=b y=y b$; if there is any such element it is called the quotient, $a$ divided by $b$, and is denoted by $\frac{a}{b}$, or $a / b$; so that

$$
a=b\left(\frac{a}{b}\right)=\left(\frac{a}{b}\right) b .
$$

The operation of finding $a / b$ when $a$ and $b$ are given is called "division ;" the element $a$ is called the "numerator," and $b$ the "denominator," of the quotient $a / b$. The special case in which the numcrator is the unit-element agrees with the definition of $1 / a$ given in theorem 14 .

Remark. From theorem 3 it is evident that if $b=\mathbb{I}$ there is no (uniquely determined) elcment $y$ such that $a=b y$; hence division by the zero-element is impossible in any system which satisfies postulate $M / 4$. On the other hand, if $b \neq \mathbb{\square}$, then $\mathbb{\square} / b=\mathbb{\square}$.

Theorem 18. [M 1, 2, 3, 5.] If the quotients in question exist, then

$$
\frac{a c}{b c}=\frac{a}{b} ;\left(\frac{a}{b}\right) \times c=\frac{a c}{b} ;\left(\frac{a}{b}\right) / c=\frac{a}{b c}: \frac{a}{b} \times \frac{c}{d}=\frac{a c}{b d} ;\left(\frac{a}{b}\right) /\left(\frac{c}{d}\right)=\frac{a d}{b c} .
$$

Moreover, if there is a unit-element, then $a / \mathfrak{l}=a$ and $a / a=\downarrow$. (Proof on p. 37.) 
Theorem 19. If the required quotients and remainders exist, then :

$$
\begin{array}{ll}
\text { (1) }[A 1 ; M 1,2,3,4,5 .] & \frac{a}{b}+\frac{c}{d}=\frac{a d+b c}{b d} ; \\
\text { (2) }[A 1,3,5 ; M 1,2,3,4,5 .] & \frac{a}{b}-\frac{c}{d}=\frac{a d-b c}{b d} .
\end{array}
$$

(Proof on p. 37.)

$$
\text { The so-culled imaginary units. }
$$

Theorem 20. $[A 1,2,3 ; M 1,2,3,4,5$.$] \quad In a system containing a$

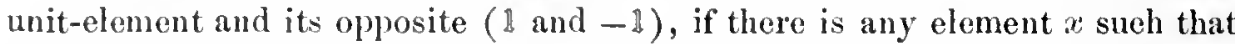
$x \times x=-1$, then there will be another element, namely $-x$, having the same property; but there cannot be more than two such elements. If there are two such elements, they are called the imagimary units (or better, the secondury units) of the system; and denoted by $i$ and $-i$; that is

$$
i \times i=-\mathbb{1} \text { and }(-i) \times(-i)=-\mathbb{1} \text {. }
$$

(Proof on p. 37.)

The term imaginary is a legacy from the eightcenth century, which has, unfortunately, become firmly fixed in mathematical literature; the elements $i$ and - $i$ are of course no more "imaginary" than any other elements which may exist in the system.*

It is a curious fact concerning these imaginary units, that no distinetion can be made between them in terms of addition and multiplication; that is, there is no true proposition concerning $i$, and expressible in terms of addition and multiplieation alone, which does not remain a true proposition when $-i$ is put in place of $i$.

\section{\$3. FURTHER DEDUCTIONS: USE OE NUMERICAL OPERATORS.}

Multiples of an element. Use of intery al numbers as coefficients.

Theorem 21, and Definition. $[A 1,2$.$] If a$ is any element of the system, then the elements

$$
a, a+u,, u+u+u, a+u+u+u, \ldots
$$

belong to the system, and are called the multiples of $\mu$. In order to secure a

* For a sketch of the history of the imaginary quantitics, see H. Hankel, loc. cit., p. 71. 
convenient notation for these successive multiples, we employ, in the manner explained below, the familiar sequence of Arabic numerals,

$$
1,2,3, \ldots ;
$$

no knowledge of these symbols is presupposed, however, beyond a rule by which, when any one of them is given, the next following one can be written down, the rule being of such a nature that each new symbol is different from all that have gone before it. Thus:

$$
\begin{aligned}
& \text { the element } a \text { is denoted by } 1 a \text {; } \\
& \text { the element } 1 a+a \text { is denoted by } 2 a \text {; } \\
& \text { the element } 2 a+a \text { is denoted by } 3 a \text {; }
\end{aligned}
$$

and so on ; in general,

$$
\text { the element } \nu,+a \text { is denoted by } \nu^{\prime} a \text {, }
$$

where $\nu^{\prime}$ is the numeral next following $\nu$. In this way the element $\mu \mu$, where $\mu$ is any Arabic numeral, is defined, and is called the $\mu^{\text {th }}$ multiple of $\alpha$.

The Arabic numerals are called, in mathematical language, the positive integral numbers, or the positive integers, and when used in the manner just described they are called coefficients; thus, in $\mu a$, the number $\mu$ is the coefficient of the element $a$.

It must be noticed that $\mu \alpha$ is not a product in the sense of postulate $M 1$, since the number $\mu$ is merely a symbol of operation and not an element of the system $a, b, c, \ldots$.In particular, the positive integral numba 1 must not be confused with the unit-element, $\mathfrak{l}$, of theorem 5 .

The statement of many theorems in regard to multiples of an element can be much simplified by the aid of the following conventions in regard to the positive integral numbers.

If $\lambda$ is any positive integer, then the integer next following $\lambda$ is called the successor of $\lambda$, and denoted by $\lambda+1$; the successor of $\lambda+1$ is denoted by $\lambda+2$; the successor of $\lambda+2$ is denoted by $\lambda+3$; and so on; in general, the successor of $\lambda+\nu$ is denoted by $\lambda+\nu^{\prime}$, where $\nu^{\prime}$ is the successor of $\nu$; that is,

$$
(\lambda+\nu)+1=\lambda+(\nu+1) .
$$

In this manner, we can define, by successive steps, a positive integer

$$
\lambda+\mu \text {, }
$$


for any two positive integer's $\lambda$ and $\mu$; this integer $\lambda+\mu$ is ealled the $s u m$, $\lambda$ plus $\mu$.

Further, if $\lambda$ is any positive integer, $\lambda+\lambda$ is denoted by $2 \lambda ; 2 \lambda+\lambda$ is denoted by $3 \lambda$; and so on; in general, $\nu \lambda+\lambda$ is denoted $\nu^{\prime} \lambda$, where $\nu^{\prime}$ is the sueeessor of $\nu$; that is,

$$
\nu \lambda+\lambda=(\nu+1) \lambda
$$

moreover, to eomplete the series, we set $1 \lambda=\lambda$.

In this manner we ean define, by suecessive steps, a positive integer

$$
\mu \lambda \text { (written also } \mu \times \lambda \text { or } \mu \cdot \lambda \text { ) }
$$

for any two positive integers $\lambda$ and $\mu$; this integer $\mu \lambda$ is called the product, $\mu$ times $\lambda$.

Finally, if $\lambda$ eomes later than $\mu$ (or $\mu$ earlier than $\lambda$ ) in the sucession of positive integers, we write $\lambda>\mu($ or $\mu<\lambda)$.

From these definitions it follows, by "mathematical induetion," that the sums and produets of the positive integral numbers obey the assoeiative, eommutative, and distributive laws for addition and multiplication, and also the laws of caneelation (proof on p. 37) ; in other words, the system of positive integers, with addition and multiplication defined as above, is itself an example of a system satisfying postulates $A 1-A 5, M 1-M 5$, so that all the definitions and theorens of $\$ 2$ can be applied to it.' Thus, the system contains a unit-element (namely the nun ber 1), but no zero-element; the remainder, $\lambda-\mu$, will exist in the system when and only when $\lambda>\mu$. The faet that the number-system satisfies the teil postulates is ineidental, however, in the present diseussion, sinee the numbers appear meeiy as symbols of operation, not is elements of the class $a, b, c, \ldots$ whose properties are primarily under consideration.

The usefulness of these definitions eoneerning the positive integral numbers is slown by the following theorems eoneerning multiples of an element of the original system:

Theorem 22. $[A 1,2$.$] If a$ be any element of the system, and $\lambda, \mu$ any positive integers, then

$$
\lambda a+\mu a=(\lambda+\mu) a \text { and } \lambda(\mu a)=(\lambda \mu) a .
$$

(Proof on p. 38.)

The first part of this theorem shows that the sum of any two multiples of $a$ is again a multiple of $a$, and that $\lambda u+\mu u=\mu u+\lambda u$; that is, the 
system of multiples of any element $a$ is a closed system with respect to addition, and obeys the commutative law. Henee we may use the notion of subtraction within this system (theorems 11-12), so that we lave, on the basis of postulates $A 1,2,3$ alone:

Theorem 23. $[A 1,2,3$.$] If \lambda>\mu$, then

(Proof on p. 39.)

$$
\lambda a-\mu a=(\lambda-\mu) a \text {. }
$$

The negative integral numbers and the zero-number.

Coneerning multiples of opposite elements, we have:

Theorem 24. $[A 1,2,3$.$] If a$ is any element whieh has an opposite, then

$$
\mu(-a)=-(\mu a),
$$

where $\mu$ is any positive integer. Henee, any element of this form may be denoted without ambiguity by $-\mu c$. (Proof on p. 39.)

This theorem suggests the use of the eomposite $\mathrm{xymbol}-\mu$ as an operator analogous to the operators already used; such a symbol $-\mu$, where $\mu$ is any positive integer, is called a negative integral number, or a negutive integer. Moreover, we may define sums and produets of positive and negative integers as follows (the purpose being to devise such definitions that the formule in theorem 22 shall remain true when $\lambda$ or $\mu$ or both are negative):

$$
\begin{gathered}
(-\lambda)+(-\mu)=-(\lambda+\mu) ; \lambda+(-\mu)=(-\mu)+\lambda=\left\{\begin{array}{c}
\lambda-\mu \text { when } \lambda>\mu, \\
-(\mu-\lambda) \text { when } \lambda<\mu ;
\end{array}\right. \\
(-\lambda) \times(-\mu)=\lambda \mu ; \quad \lambda \times(-\mu)=(-\lambda) \times \mu=-(\lambda \mu) .
\end{gathered}
$$

These definitions are only partially satisfaetory, however, sinee there is no meaning attaehed to a sum of the form $\lambda+(-\lambda)$. To obviate this difficulty, we introduce a new operator ealled the zero-number, 0 , with the eonvention that

$$
0 \varkappa=\mathbb{1}
$$

for every element $\alpha$, and the following definitions as to sums and products :

$\lambda+(-\lambda)=0 ; \lambda+0=0+\lambda=\lambda ;(-\lambda)+0=0+(-\lambda)=-\lambda ; 0+0=0 ;$

$$
\lambda \times 0=0 \times \lambda=0 ; \quad(-\lambda) \times 0=0 \times(-\lambda)=0 ; 0 \times 0=0 .
$$

This zero-number, 0 , which is merely an operator, must be carefully distinguished from the zero-element, $\mathbb{\|}$, of theorem 1 , 
The positive and negative integers, together with the zero-number, make up the system of all integral mumbers. The system of all integral numbers is, incidentally, another example of a system uhich satisfies, sll the postulates $A 1-A 5$, M1-M5; it contitins a unit-clement (the number 1), and a zero-element (the number 0) ; und subtraction is always possible. The usefulness of these definitions is shown by the following theorem:

Theorem 25. [A1, 2, 3.] If " is any elenent which has an opposite, then the formule of theorem 22 loold true when $\lambda$ and $\mu$ are any integral numbers (positive, negative or zero).

Submultiples and rational fructions of an element. Use of the rational numbers as coefficients.

The following theorems depend on the postulate of non-circularity $(A 4)$, which has not hitherto been recunired.

Theorem 26 . $\quad[A 1,2,3,4$.$] \quad If \mu a=\mathbb{I}$ (where $\mu$ is any positive integer), then $a=\mathbb{a}$. In other words, if any multiple of $a$ is the zero-clement, then $"$ itself is the zero-element. (Proof on p. 39.)

Corollery. If $\lambda \neq \mu$, and $a \neq \mathbb{0}$, then $\lambda a \neq \mu a$. In other words, if $a$ is not the zero-element, then every multiple of a is different from every other multiple of " ; this justilies the name "lnw of non-cireularity" proposed for postulate $A 4$.

In view of this theorem, we notice that every system which satisfies postulates A1-At, and contains more than a single element, must be inf nite.

Theorem 27 , and Definition. $[A 1,2,3,4$.$] If \alpha$ is any element, and $\mu$ any positive integer, then there cannot be nore than one element $x$ such that $\mu x=a$; if there is any such element, it is called the $\mu^{\text {th }}$ submultiple of $a$, and denoted by $\frac{a}{\mu}$, or $a / \mu$ : that is,

$$
\mu\left(\frac{\prime \prime}{\mu}\right)=a .
$$

In partieular, $a / 1=a$.

Corollary. If $\lambda \neq \mu$, and $a \neq \mathbb{I}$, then $a / \lambda \neq u / \mu$.

Theorem 28, and Definition. [A1, 2, 3, 4.] If $a / \mu$ exists, then

$$
\lambda\left(\frac{u}{\mu}\right)=\frac{\lambda a}{\mu},
$$


where $\lambda$ and $\mu$ are any positive integers. Any element of this form is ealled a retional fraction of $u$, aud may be denoted without ambiguity by $\frac{\lambda}{\mu} a$. In partieular, $\frac{\lambda}{1} \iota=\lambda u$, and $\frac{1}{\mu} u=\frac{a}{\mu}$. (Proof on p.39.)

Corollary. If $\lambda \mu_{1}=\mu \lambda_{1}$, then $\frac{\lambda}{\mu} a=\frac{\lambda_{1}}{\mu_{1}} a$.

This theorem 28 suggests the use of the composite symbol $\frac{\lambda}{\mu}$ as an operator analogous to the operators already used; such a symbol $\frac{\lambda}{\mu}$, where $\lambda$ and $\mu$ are any positive integers, is ealled a positive rational number.

In order to make these symbols as useful as possible, we agree, in the first plaee, to set $\frac{\lambda}{1}=\lambda$, and to eall $\frac{\lambda}{\mu}=\frac{\lambda_{1}}{\mu_{1}}$ wherever $\lambda \mu_{1}=\mu \lambda_{1}$; with this convention, if $\xi$ and $\eta$ are any positive rational numbers, we shall have $\xi a=\eta a$ whenever $\xi=\eta$.

Further, we define the sum and prodnet of two positive rational numbers by the formula

$$
\frac{\lambda}{\mu}+\frac{\lambda_{1}}{\mu_{1}}=\frac{\lambda \mu_{1}+\mu \lambda_{1}}{\mu \mu_{1}}, \quad \frac{\lambda}{\mu} \times \frac{\lambda_{1}}{\mu_{1}}=\frac{\lambda \lambda_{1}}{\mu \mu_{1}},
$$

these definitions reducing to the previous definitions for positive integers when $\mu=\mu_{1}=1$.

Finally, we agree to write $\frac{\lambda}{\mu}>\frac{\lambda_{1}}{\mu_{1}}$ whenever $\lambda \mu_{1}>\mu \lambda_{1}$.

With these definitions of addition and multiplication, the system of positive rational numbers is, incidentally, a system which sutisfies all the postulates $A 1-A 5, M 1-1 / 5$; it eontains a unit-element (the number 1), but no zeroelement; the remainder, $\xi-\eta$, exists in the system when and only when $\xi>\eta$; but division is always possible.

The usefulness of these definitions is apparent from the following theorems (compare theorems 22-23):

Theorem 29. $[A 1,2,3,4$.$] If a$ be any element all of whose submultiples exist, and if $\xi, \eta$ are any positive rational number's, then

$$
\xi a+\eta a=(\xi+\eta) \iota \text { and } \xi(\eta(\iota)=(\xi \eta) a \text {. }
$$

The first part of this theorem shows that the sum of any two rational fraetions of $u$ is again a rational fraetion of $a$, and that $\xi u+\eta a=\eta \alpha+\xi a$; that is, the system of rational fraetions of any element $u$ is a elosed system 
with respeet to addition, and obeys the commutative law $(A 5)$. Henee we may use the notion of subtraction within this system (see theorems 11-12), so that we have, on the basis of postulates $A 1,2,3,4$ alone:

Theorem 30. $[A 1,2,3,4$.$] If a$ is an element all of whose submultiples exist, and if $\xi, \eta$ are positive rational numbers sueh that $\xi>\eta$, then $\xi u-\eta \iota=(\xi-\eta) \propto$.

The nequtive rational numbers.

Conceruing lational fraetions of opposite elements we have :

Theorem 31. $[A 1,2,3,4$.$] lf a$ is any element whieh has an opposite and all its submultiples, then

$$
\frac{\lambda}{\mu}(-a)=-\left(\begin{array}{l}
\lambda \\
\mu
\end{array}\right)
$$

Where $\frac{\lambda}{\mu}$ in ang; ponitive rational number. Hence, any element of this form niay be denoted withont ambignity by $-\frac{\lambda}{\mu} a$.

This theorem suggests that we add the symbol $-\frac{\lambda}{\mu}$ to our list of operator's; sueh a symbol $-\frac{\lambda}{\mu}$, where $\lambda$ and $\mu$ are any positive integers, is ealled a ne gatice rational number. The negative rational numbers bear the same relation to the positive rational numbers that the negative integers bear to the positive integers. The positive and negative rational numbers, together with the zero-number, constitute the set of all rational numbers, and the sum and product of any two rational numbers are defined by precisely the same eonventions as in the ease of all integer's (page 17.)

The system of all rational numbers, with addition and nultiplication defined in this uay, is wtill another example of a system which satisfies all the postulates Al-A5, $M 1-1 / 5$; this system contains a unit-element (the number 1 ), and a zero-element (the number 0 ); every element has an opposite, and every element except zero has a reciproeal, so that subtraction and division are always possible, except division by zero.

The usefulness of these definitions is shown by

Theorem 32. $[A 1,2,3,4$.$] If a$ is any element whieh has an opposite and all its submultiples, then the formulie of theorem 22 hold true when $\lambda$ and $\mu$ are replaced by any rational number's (positive, negative, or zero). 
Further theorems on the use of numerical coefficients.

Theorem 33. [A1,2;M 1, 4.] If $\lambda, \mu$ are any positive integers, and $a, b$ any elements, then

$$
(\lambda a)(\mu b)=(\lambda \mu) a b .
$$

Theorem 34. $[A 1,2,3 ; M 1,4$.$] \quad If \xi, \eta$ are any integral numbers, and $a, b$ any elements whieh have olposites, then

$$
(\xi a)(\eta b)=(\xi \eta) a b .
$$

Theorem 35. $[A 1,2,3,4 ; M 1,4$.$] If \xi, \eta$ are any rational nunbers, and $a, b$ any clements which have opposites and submultiples, then

$$
(\xi a)(\eta b)=(\xi \eta) a b .
$$

(Proofs on p. 39.)

To avoid further repetition, the following theorens are stated at onee for the general case, in which $\xi, \eta$ and $\theta$ stand for any rational numbers, and $a$, $b, x, y$ for any elements which have opposites and submultiples:

Theorem 36. [A1, 2, 3, 4.] If $\theta x=\theta y$ and $\theta \neq 0$, then $x=y$; and if $\xi a=\eta a$ and $a \neq \mathbb{I}$, then $\xi=\eta$. (Proof on p. 40.)

Theorem 37. $[A 1,2,3,4$.$] If \xi a=\mathbb{l}$, then either $\xi=0$ or $a=\mathbb{I}$. (By theorem 3b.)

Theorem 38. $[A 1,2,3,4,5.] \xi(a+b)=\xi a+\xi b$. (Proof on 1) 40.)

Theorem 39. $[A 1,2,3,4,5.] \xi(a-b)=\xi a-\xi l$. (Proof on p. 40.)

Theorem 40. $[A 1,2,3,4 ; M 1,2,3,4,5$.$] \quad If \eta \neq 0$ and $b \neq \mathbb{\mathbb { T }}$, then

$$
\frac{\xi a}{\eta b}=\left(\frac{\xi}{\eta}\right)_{b}^{a}
$$

(Proof on p. 41.)

$$
\text { Powers of an element. }
$$

Use of the integral numbers as exponents.

Theorem 41, and Definition. $[M 1,2$.$] If a$ is any element of the system, then the elements

$$
a, \quad a \times a, \quad a \times a \times a, \quad a \times a \times a \times a, \cdots
$$


belong to the system and are called the powers of $a$.

The element $\quad a$ is denoted by $a^{1}$; the elenent $a^{1} \times u$ is denoted by $a^{2}$; the element $a^{2} \times a$ is denoted by $a^{3}$;

and so on ; in general,

tine element $a^{\nu} \times a$ is denoted by $a^{\nu^{\prime}}$,

where $\nu^{\prime}$ is the positive integer next following $\nu$. In this way the element $a^{\mu}$, where $\mu$ is any positive integer, is defined, and is ealled the $\mu^{\text {th }}$ power of $a$.

The positive integers when used in this manner are ealled exponents; thus, ' in $a^{\mu}$, the positive integer $\mu$ is the exponent of the elenent $a$.

By the use of the definitions for the sum and product of two positive integers, we have:

Theorem 42. [M1, 2.] If $a$ is any element of the system, then

$$
a^{\lambda} \times a^{\mu}=a^{\lambda+\mu} \text { and }\left(a^{\lambda}\right)^{\mu}=a^{\lambda \mu},
$$

when $\lambda, \mu$ are any positive integral numbers (compare theorem 22).*

The first part of this theorem shows that the produet of any two powers of $a$ is again a power of $u^{\prime}$, and that $a^{\lambda} \times a^{*}=a^{\mu} \times a^{\lambda}$; that is, the system of porers of any element $a$ is a closed system with respect to multiplication, and obeys the commutatice law. Hence we may use the notion of division within this system (theorems 17-18), so that we have, on the basis of postulates M1, 2, 3 alone:

Theorem 43. $[1 / 1,2,3$.$] If \lambda>\mu$, then $a^{\lambda} / a^{\mu}=a^{\lambda-\mu}$. (Compare theorem 23).

Concerning powers of reciprocal elenents, we have:

Theorem 44. $[M 1,2,3$.$] \quad If a$ is any element which has a reeiproeal, then

$$
\left(\frac{\mathbb{l}}{a}\right)^{\mu}=\frac{\mathbb{l}}{a^{\mu}} \text {. }
$$

where $\mu$ is any positive integer.

These theorems suggest the use of the negative integral numbers and the zero-number as exponeuts, operating on any element which has a reciprocal; thus; if we agree to define

* The proofs of theorems $41-45$ are similar to the proofs of theorems $21-25$, and may well be left to the reader. 


$$
\prime^{-\mu}=\frac{\mathfrak{l}}{\prime^{\mu}} \text { and } \quad \prime^{0}=\mathbb{1},
$$

we shall have:

Theorem 45. $[M 1,2,3$.$] If a$ is any element which has a reciprocal, then the formule of theorem 42 hold true when $\lambda$ and $\mu$ are any integral numbers (positive, negative, or zero).

\section{On the use of rational exponents.}

The analogy between these theorems 41-45 on powers and the theorems 21-25 on multiples suggests the possibility of carrying the parallel one step further and introducing also the use of rational numbers as exponents. The attempt to do this is eomplieated, however, by the faet that we have, in general, no law for multiplication eorresponding to the law of non-cireularity for addition (see theorem 26 ); that is, if $a$ and $\mu$ are given, there may well be more than one element $x$ such that $x^{\mu}=a$. If, therefore, we define $a^{1 / \mu}$ to signify an element $x$ such that $x^{\mu}=\alpha$, we must understand that we are introducing a symbol whose value is, in general, not uniquely determined. Such nultiple-valued symbols ean indeed be used, as is well known, to good advantage, and their properties can be made to conform, approximately, to the laws of theoren 42 ; but the study of them would earry us beyond the limits of the present paper.

\section{Further theorems on the use of numerical exponents.}

Theorem 46. $[M 1,2,3,5$.$] If the requisite reciprocals and quotients$ exist, then

$$
(a b)^{\mu}=\iota^{\mu} b^{\mu} \quad \text { and } \quad\left(\begin{array}{l}
a \\
b
\end{array}\right)^{\mu}=\frac{r^{\mu}}{b^{\mu}}, \quad .
$$

when $\mu$ is any integer (positive, negative, or zero).

Sinee the rational numbers form. a system which satisfies the postulates $A 1-A 5, M 1-M 5$ with respeet to their addition and multiplication, the definitions of $a^{\mu}, a^{-\mu}$, and $a^{0}$ will apply equally when the elenent $a$ is replaced by any rational number $\xi$ (provided $\xi \neq 0$ in $\xi^{-\mu}$ ). Using this notation we have:

Theorem 47. $[A 1,2,3,4 ; M 1,2,3,4$.$] If the requisite reciprocals$ exist, then

$$
\left(\xi^{\prime \prime}\right)^{\mu}=\left(\xi^{\mu}\right) \iota^{\mu},
$$


when $\mu$ is any integer, and $\xi$ any rational number (provided $\xi \neq 0$ when $\mu$ is negative).

Concerning a power of a sum of two terms, we have the following inportant theorem, known as the binomial theorem for positive integral exponents:

Theorem 48. $[A 1,2 ; M 1,2,4,5$.$] If \mu$ is any positive integer, then $(a+b)^{\mu}=t^{\mu}+\frac{\mu}{1} a^{\mu-1} b+\frac{\mu(\mu-1)}{1 \cdot 2},^{\mu-2} b^{2}$

(Proof by induction.)

$$
+\frac{\mu(\mu-2)(\mu-3)}{1 \cdot 2 \cdot 3} \iota^{\mu-3} b^{3}+\cdots+b^{\mu} .
$$

Concerning the subtraction of two powers, we have:

Theorem 49. $[A 1,2,3,5 ; M 1,2,4,5$.$] \quad If a-b$ exists, then

$$
a^{\mu+1}-b^{\mu+1}=(a-b)\left(a^{\mu}+a^{\mu-1} b+a^{\mu-2} b^{2}+\cdots+a b^{u-1}+b^{\mu}\right),
$$

where $\mu$ is any positive integer.

$$
\text { Equations of the } \mu^{\text {th }} \text { degree in } x \text {. }
$$

A conditional equation of the form

$$
c_{0} x^{\mu}+c_{1} x^{\mu-1}+c_{2} x^{\mu-2}+\cdots+c_{\mu-1} x+c_{\mu}=\mathbb{I},
$$

where $\mu$ is any given positive integer, and $c_{0}(\neq \mathbb{I}), c_{1}, c_{2}, \cdots, c_{\mu}$ are any elements of the system, is called an equation of the $\mu^{\text {th }}$ degree in $x$ : any element $x$ which satisfies the condition is called a root of the equation; the left-hand side of the ecjuation is called a polynomial of the $\mu^{\text {th }}$ degree in $x$; and the given elements $c_{0}, c_{1}, \ldots, c_{\mu}$ are called the coefficients of the polynomial (or of the equation.)

Lemma. If $x=r$ is a root of an equation of the $\mu^{\text {th }}$ degree in $x$, then the equation can be written in the form

$$
(x-a) \times(\text { a polynomial of next lower degree in } x)=\mathbb{a} \text {, }
$$

provided the system is one in which subtraction and division (except division by ir) are always possible.

Theorem 50. $[A 1,2,3,4,5 ; M 1,2,3,4,5$.$] \quad If the system is one$ in which every equation of the $\mu^{\text {th }}$ degree has at least one root, then every such equation can be written in the form

$$
\left(x-a_{1}\right)\left(x-a_{2}\right)\left(x-a_{3}\right) \cdots\left(x-a_{\mu}\right)=\emptyset,
$$


Eaeh of the elements $a_{1}, a_{2}, \ldots a_{\mu}$ will be a root of the equation, and the equation eannot have any other roots. (Proof by successive applications of the lemma.)

Redundancy of the commutative law for addition.

It remains to prove, as stated above, that the commutative law for addition (postulate $A 5$ ) is redundant when the list of postulates is taken as a whole.

Theorem. The eommutative law for addition,

$$
a+b=b+a,
$$

is a eonsequenee of postulates $A 1,2,3 ; M 1,3_{1}$ or $3_{2}, 4$.

This theorem was given first by H. Hankel in 1867; the proof is here modified so as not to require the existenee of a unit-element.

Proof. Let $c$ be any element diflerent from $\mathbb{a}$ (by postulate I). Then

$$
(a+b)(c+c)=(a+b) c+(a+b) c=a c+b c+a c+b c, \quad \text { by } M 4_{1} \text { and } M 4_{2} \text {; }
$$

but also

$$
(a+b)(c+c)=a(c+c)+b(c+c)=a c+a c+b c+b c, \quad \text { by } M 4_{2} \text { and } M 4_{1} ;
$$

henee

and therefore

$$
\begin{aligned}
b c+a c+b c & =a c+b c+b c, & & \text { by } A 3_{1}, \\
b c+a c & =a c+b c, & & \text { by } A 3_{2} .
\end{aligned}
$$

Henee

and therefore

$$
(b+a) c=(a+b) c, \quad \text { by } M 4_{2},
$$

$$
b+a=a+b, \quad \text { by } M 3_{2} .
$$

In order to use $M 3_{1}$ instead of $M 3_{2}$ in the proof, we should have merely to start with $(c+c)(a+b)$ instead of $(a+b)(c+c)$.

\section{\$4. SPECial LAWS OF ADDition aNd MULtiplication.}

PARTICULAR TYPES OF ELEMENTARY ALGEBRA.

The postulates $A 1-A 5, M 1-M 5$ may be satisfied, as we have seen, by many different systems; for example, the system of positive integers, with addition and multiplieation defined as on page 15, or the system of all rational numbers, with addition and multiplication defined as on page 20 . 
Two systems satisfying these general laws are said to be isomorphic with respect to addition and multiplieation when the following eonditions are satistied :

1) the elements of the two systems ean be brought into one-to-one correspondence (so that eaeh element of one elass is paired with one and only one element of the other elass, and reciproeally eaeh element of the seeond elass is paired with one and only one element of the first elass); and

2) this eorrespondenee ean be set up in sueh a way that whenever $a$ and $b$ in one class correspond to ${ }^{\prime}$ and $b^{\prime}$ in the other elass, then $a+b$ will correspond to $a^{\prime}+b^{\prime}$, and $a \times b$ will correspond to $a^{\prime} \times b^{\prime}$.

Two systems satisfying the general laws of $\$ 1$, and isomorphie with eaeh other, are said to belong to the same type of algebra; two systems satisfying the same general laws, but not isomorphic with each other are said to belong to different types of algebre. The various systems of numbers employed as operators in $\$ 2$ afford exmples of several diflerent types of algebra.

Two algebras of the same type are formally identicul as far as addition and multiplieation are eoneerned; that is, they eannot be distinguished by any properties expressible in terms of addition and multiplieation alone.

The set of postulates $A 1-A 5, M 1-M 5$ is elearly not suffieient to determine any one type of algebra, sinee all these postulates ean be satisfied by various systems, non-isomorphie with one another. In order to obtain, for each of the more important types of elementary algebra, a set of postulates whieh shall completely determine that type, we add eertain further postulates, given in the present section. Euch of the resulting sets of postulates determines completely one type of algebra, in the sense that any two systems whieh satisfy all the postulates of that set will be isomorphic with respect to addition and multiplication.

A set of postulates whieh is suffieient to determine a partieular type of system in this manner has been called a calegorical set of postulates.* ${ }^{*}$ This

* The earliest set of postulates having this elaracter is probably the set of five postulates for the system of natural unmbers with respeet to snecession, given by G. Peano in 1891 . [Rivistı di Matematica, vol. 1 (1891), p. 87; Formulaire de Mathématiques, vol. 2 (1898), p. 2.] Other sets of postulates of the same kind, for the systems of positive real, positive rational, and positive integral nmmbers with respeet to addition, were given by the present writer in 1902. [Trans. Amer. Math. Soc., vol. 3, pp. 264-284, espeeially theorems II, II', 111", on pp. 277, 282, 283. See also ibid., vol. 6 (1905), p. 41.] The name eategorieal was introdueed in 1904 by 0 . Veblen, who has unade important use of the notion in his sets of postulates for geometry. [Trans. Amer. Math. Soc., vol. 5 (1904), p. 346.] 
name has been eritieised by Couturat as inappropriate; ; but whether or not the name has been happily ehosen, the notion itself is of fundamental importanee. Any eategorieal set of postulates ineludes, by implieation, all the properties of the type of system which it determines, as far as they eoncern the operations in question ; thus, in ease of a eategorieal set of postulates for a type of algebra every proposition which is expressible in terms of addition and multiplication alone must either be a eonsequenee of the postulates of sueh a set, or else be in contradietion with them. This is not true of a noneategorieal set of postulates, like the set $A 1-A 5, M 1-M 5$; for example, the proposition "there is an element $z$ in the system, sueh that $z+z=z$ " is neither dedueible from these postulates nor in eontradietion with them; it is true in some systems whieh satisfy the postulates, and false in others.

The olject of the present section is, then, to give a sufficient, or categorical, set of postulates for each of the types of algebra here considered. (Other types of algebra - like the algebra of all real numbers, or the algebra of all complex numbers-require for their eharaeterisation properties which involve the notion of order, and are therefore not diseussed in the present paper.)

The new postulates all coneern the existence, in the system, of elements satisfying eertain eonditions, and are therefore designated by the letter $E$.

The algebra of positive integers and the algebra of positive integers with zero.

The first of the speeial laws whieh we add to the general laws of $\S 1$ is the following:

Postulate E1. There is a qnit-element in the system (see theoreme 5).

All the multiples of this unit-element will exist in the system, by postulates $A 1,2$, and may be ealled the positive integral elements of the system. By theorems 22 and 33, these positive integral elements form a elosed system with respect to addition and multiplication (see postulates $A 1$ and $M 1$ ); henee, to obtain a suffieient, or categorieal, set of postulates for this type of algcbra, we have only to add the following postulate:

Postulate $F$. Thereare no elements in the system besides those required by the other postulates.

* L. Couturat, Les principes des Mathématiques, 1905, p. 169. 
That is, the algebre of positive integers is completely determined by postulates

$$
\text { A1, 2; } M 1,2,3,4 ; E 1 ; F .
$$

Every system which satisfies these eight conditions will be formally identical with the system of positive integers, as far as addition and multiplication are concerned. (The other postnlates of $\$ 1$ become redundant after $E 1$ and $F$ are added.)

Further, if we add

Postulate E2. There is a aro-element in the system (see theorem 1), then the postulates

$$
A 1,2,3 ; M 1,2,3,4 ; E 1,2 ; F
$$

completely detcrmine the algetru of positive integers with zero. Evcry system which satisfics these ten conditions will be formally identical with the system of positive integer's with zero, as far as addition and multiptication are concerned,

This postulate $F$ may be called, for lack of a better name, the law of non-superfluity." The "other postulatcs" referred to mcan, of course, in each casc, the other postulates of the set considered in that case.

The algebra of all integers.

Besides postulates $E 1$ and $E 2$ we may add also

Postulate E3. The opposite of the unit-element exists in the system (see theorem 8).

When this postulate is added, the system will contain $\mathbb{1}, \mathbb{1}$, and $-\mathbb{1}$, and all the multiples of $\mathbb{1}$ and $-\mathbb{1}$; thesc elements form a closed system with respect to addition and nultiplieation (by theorems 25 and 34), and may be called the integural elements of the system.

Hence the algebra of all integer's is completely determined by postulates

$$
A 1,2, \ddot{3} ; M 1,2,3,4 ; E 1,2,3 ; F \text {. }
$$

*This postulate is mnch less vague than Hilbert's "Axiom of Completeness" (Axiom der Vollsiändigkeit), whicls is appirently intended to serve a sinilar purposc. [See Jahresbericht der Deulschon Wuthemuliker-Vreivigung, vol. 8 (19011), part 1, p. 184] Hilbert does not use the notion of isımorphisu, huwever, and his "Axions of geonetry," as a matter of fact, do not form a categurical set. 
Every system which satisfies these eleven conditions will be isomorphic with the system of all integers, with respect to addition and multiplieation.

The algebra of positive rationals, and the algebra of positive rationals with zero.

We now introduce another postulate,

Postulate E4. All the submultiples of the unit-element exist in the system.

By virtue of this postulate, all the rational fractions of the unit-clement (theorem 28) will exist in the systen, and may be called the posilive rational elements of the system. Moreover, these elements form a elosed system with respect to addition and inultiplication, by theorems 29 and 35 .

Hence, the algebra of the positive rationals is completely determined by postulates

$$
A 1,2,3,4 ; M 1,2,3,4 ; E 1,4 ; F \text {. }
$$

The isomorphism of any two systems which satisfy these eleven conditions is established by means of the fact that the sum and product of two elements

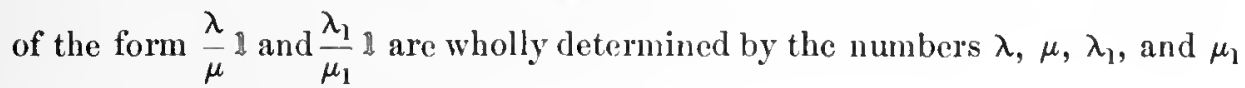
(see theorems 29 and 35 ).

Similarly, the algebra of positive rationals with zero is completely determined by the postulates

$$
A 1,2,3,4 ; M 1,2,3,4 ; E 1,2,4 ; F \text {. }
$$

Here, and below, postulate $M 5$ becomes redundant when the later postulates are added.

$$
\text { The algebra of all rationals. }
$$

The algebra of all rationals (positive, negative, or zero) is eompletely determined by postulates

$$
A 1,2,3,4 ; M 1,2,3,4 ; E 1,2,3,4 ; F .
$$

Every system which satisfies these thirteen conditions will be formally identical with the system of all rational numbers, with respect to addition and multiplication. This type of algebra is the simplest type in which the four operations of addition, multiplícation, subtraction, and division (except division by zero) are always possible. 
It will be noticed that in all the types of algebra so far considered, the isomorphism between two systems of the same type can be set up in only one way, since the unit-elenents of the two systems must be made to correspond.

\section{The algebras of complex quantities.}

We now consider postulates $E 1,2,3$, with

Postulate E5. There is a pair of imaginary units in the system (see theorem 20).

An imaginary unit, $i$, defined by the equation $i^{2}=-1$, cannot be an integral or rational element of the system, because if it were, then $i^{2}$ could not be -1 . Ilence, by $A 1$, the addition of this postulate $E 5$ introduces a new class of elements of the form $\xi \mathbb{d}+\eta i$, where $\xi$ and $\eta$ are integral or rational numbers. Elements of this form are called complex elements of the system, with integral or with rational coefficients. No further elements are introduced by unttiplication, however, since

$$
(\xi \mathbb{d}+\eta i)\left(\xi_{1} \mathbb{A}+\eta_{1} i\right)=\left(\xi \xi_{1}-\eta \eta_{1}\right) \mathbb{1}+\left(\xi \eta_{1}+\eta \xi_{1}\right) i .
$$

Hence a definite type of algebra, which may be called the algebra of complex quntities with integral coefficients is completely determined by postulates

$$
A 1,2,3 ; M 1,2,3,4 ; E 1,2,3,5 ; F \text {. }
$$

In a similar way another type of algebra, called the algebra of complex quantities with rational coefficients is completely determined by postulates

$$
A 1,2,3,4 ; M 1,2,3,4 ; E 1,2,3,4,5 ; F \text {. }
$$

It will be noticed that in the ease of either of the complex algebras, the isomorphism between two systems of either type can be set up in two ways, on account of the ambiguity in the choice of the element $i$ (see theorem 20).

An example of a system which satisfies all the postulates for the algebra of complex quantities with integral [or rational] coefficients is the class of all ordered pairs of integral [or rational] numbers, $(\xi, \eta)$, with addition and multiplication defined as follows:

$$
\begin{gathered}
\left(\xi_{1}, \eta_{1}\right)+\left(\xi_{2}, \eta_{2}\right)=\left(\xi_{1}+\xi_{2}, \eta_{1}+\eta_{2}\right), \\
\left(\xi_{1}, \eta_{1}\right) \times\left(\xi_{2}, \eta_{2}\right)=\left(\xi_{1} \xi_{2}-\eta_{1} \eta_{2}, \xi_{1} \eta_{2}+\eta_{1} \xi_{2}\right) .
\end{gathered}
$$

Here $\mathbb{\mathbb { N }}=(0,0), \mathbb{A}=(1,0), i=(0,1)$ or $(0,-1)$, and $(\xi, \eta)=\xi \mathbb{1}+\eta \dot{ }$. The system thus constructed is called the system of orlinary complex numbers with integral [or rational] coefficients; the construction of some example of 
this kind is necessary to establish the consistency of the last two sets of postulates.

\section{The algebra of all algebraic quantities.}

Any equation of the $\mu^{\text {th }}$ degree in $x$, in which the coefficients are integral elements of the system, may be written in the form

$$
\lambda_{0} x^{\mu}+\lambda_{1} x^{\mu-1}+\lambda_{2} x^{\mu-2}+\cdots+\lambda_{\mu-1} x+\lambda_{\mu} \cdot \mathbb{1}=\mathbb{u},
$$

where $\lambda_{0}(\neq 0), \lambda_{1}, \cdots \lambda_{\mu}$ are integral numbers; such an equation is called an algebraic equation with integral coefficients, and any root of such an equation is called an algelraic element of the system.

It is easy to show that if $x$ and $y$ are algebraic elcnents, then $x+y$ and $x y$ are also algebraic elements; that is, the algebraie elements of a system form a closed system with respect to addition and multiplication.* ${ }^{*}$.

Further, if the coefficients in any equation of the form

$$
c_{0} x^{\mu}+c_{1} x^{\mu-1}+c_{2} x^{\mu-2}+\cdots+c_{\mu-1} x+c_{\mu} \cdot \mathbb{1}=\mathbb{I}
$$

are algebraic elements, then all the roots of such an equation (in so far as they exist in the system) are also algebraic elements. *

Hence, in order to obtain a systen in which every such equation las a root, we need to add merely the following postulate:

Postulate E6. Every algebraic equation of the $\mu^{\text {th }}$ degree with integral coefficients has at least one root.

Then the postulates

$$
A 1,2,3,4 ; M 1,2,3,4,5 ; E 1,2,6 ; H
$$

determine completely a type of algebra called the algebrct of all algebraic quentities. All the other types of algebra which have bcen considered.in this section are sub-algebras within this algebra of algebraic quantities.

In this algebra, the opposite of the unit-element, and all the submultiples of the unit-element, exist (since the equations $x+\mathbb{1}=\mathbb{1}$ and $\lambda x-\mathbb{1}=\mathbb{1}$ lave roots in the system); moreover, there is a pair of inaginary units, namely,

* For the proofs of these theorems, which are due to R. Dedekind (1877), and involve merely an elementary knowledge of determinants, the reader is referred to $P$. Baehmann's Zahlentheorie, vol. 5 (Allgemeine Arithmetik der Zahlenkarper, 1905), pp. 3-6. Another method of proof, depending on elementary properties of symmetric funetious, is given in Borel and Drach's Théorie des nombres et l'algèbre supérieure, 1895, p. 184. 
the roots of the equation $x^{2}+\mathbb{b}=\mathbb{1}$. Henee all the postulates $E 1-E 6$ are satisfied.

This type of algebra is the simplest type in which the operations of addition, multiplication, subtraction, and dirision (exeept division by the zeroelement) are always possible, and in which every equation of the $\mu^{\text {th }}$ degree in $x$ (the eoefficients being any elenents of the system) always has a root; it therefore forms a suitable stopping-place for the discussion in the present paper.

The problem of constrneting an example of a system of this type is an interesting one, into whieh we eamnot enter here ; ${ }^{*}$ the consistency of the postulates is usually established by the fact that the algebra of algebraic quantities is a sub-algebra within the algebra of veetors described in the introduction.

§5. EXAMPLES OF sYSTEMS WIICII SATISEY SOME BUT NOT ALL OF THE GLXERAL LAWS OF $\$ 1$. PROOFS OF INDEPENDENCE.

In this section we establish the independence of the postulates $A 1-A 4$, $M /-1 / 5$, by exhibiting, in the ease of each of the postulates, a system whieh satisfies all the other postulates, but not the one for whieh it is numbered. No one of the postulates, therefore, is dedueible from the remaining postulates; for, if it were, then any system whieh possessed all the othor properties would possess this property also, which, as the examples show, is not the case.

'The rules of eombination in these pseudo-algebraie systems we denote by $\oplus$ and $\odot$, reserving the symbols + and $\times$ for use between numbers, in the sense explained in $\$ 3$. In describing each system, we must give: (1) the class of elements eonsidercd, (2) the rule of eombination ealled $\oplus$, and (3) the rule of combination called $\odot$.

\section{List of the postulates of $\$ 1$ (general laws).}

A1. $a \oplus b$ in the system.

A2. $(a \oplus b) \oplus c=a \oplus(b \oplus c)$.

A3. (1) If $a \oplus x=a \oplus y$, then $x=y$.

(2) If $x \oplus a=y \oplus a$, then $x=y$.

A4. If $\mu x=\mu y$, then $x=y$.

[A5. $\quad a \oplus b=b \oplus a$.

* See E. Borel and J. Drach, Thérrie des nombres et l'alyèbre supérieure, 1895, p. 157. For an early statement of the problem. compare H. Hankel, loc. cit., 1867, $\$ 12$. 
M1. $a \odot b$ in the system.

M2. $(a \odot b) \odot c=a \odot(b \odot c)$.

M3. (1) If $a \odot x=a \odot y$ and $a \oplus a \neq a$, then $x=y$.

(2) If $x \odot a=y \odot a$ and $a \oplus a \neq a$, then $x=y$.

M4. (1) $a \odot(b \oplus c)=(a \odot b) \oplus(a \odot c)$. (2) $(b \oplus c) \odot a=(b \odot a) \oplus(c \odot a)$.

M5. $a \odot b=b \odot a$.

\section{Examples of pseudo-algebras.}

The examples whieh prove the independence of the several postulates are the following, all of which are eonstructed out of numerical elasses with which the reader has already been made familiar in $\$ 3$.

All except $A 1$. The elass of all rational numbers, with $\oplus$ and $\odot$ defined as follows : $a \oplus b=a+b$ whenever $a+b=0$; otherwise, $a \oplus b$ not in the class. $a \odot b=a b$, where $a b$ denotes the ordinary produet.

$A l l$ except $A 2$. The elass of all rational numbers. $a \oplus b=2(a+b)$. $a \odot b=a b$.

All except $A 3_{1}$ and $A 5$. The elass of positive rational numbers. $a \oplus b=a . \quad a \odot b=a b$.

All except $A 3_{2}$ and $A 5$. The elass of positive rational numbers. $a \oplus b=b . \quad a \odot b=a b$.

All except $A 3_{1}$ and $A 3_{2}$. The elass of all rational numbers. $a \oplus a=a$ but if $a \neq b$, then $a \oplus b=0 . \quad a \odot b=a b$.

Ail except $A 4$. A class eonsisting of nine elements, $0,1,2, \ldots, 8$, with $\oplus$ and $\odot$ defined by means of the following tables :

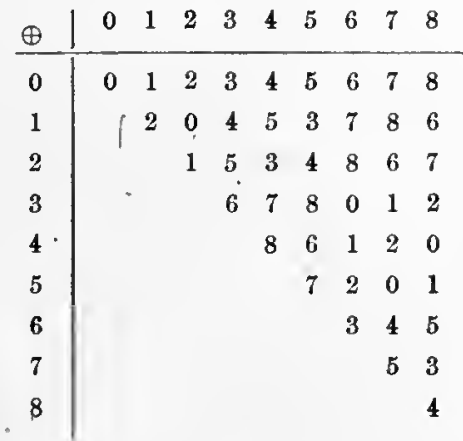

For example, $3 \oplus 7=7 \oplus 3=1 ; \quad 3 \odot 7=7 \odot 3=2$

\begin{tabular}{l|lllllllll}
$\odot$ & 0 & 1 & 2 & 3 & 4 & 5 & 6 & 7 & 8 \\
\hline 0 & 0 & 0 & 0 & 0 & 0 & 0 & 0 & 0 & 0 \\
1 & & 1 & 2 & 3 & 4 & 5 & 6 & 7 & 8 \\
2 & & & 1 & 6 & 8 & 7 & 3 & 5 & 4 \\
3 & & & & 4 & 7 & 1 & 8 & 2 & 5 \\
4 & & & & & 2 & 3 & 5 & 6 & 1 \\
5 & & & & & & 8 & 2 & 4 & 6 \\
6 & & & & & & & 4 & 1 & 7 \\
7 & & & & & & & & 8 & 3 \\
8 & & & & & & & & & 2
\end{tabular}

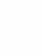


This system (whieh is a Galois Field of order $3^{2}$ ) satisfies also all the existenee postulates $E 1,2,3,4,5$. Thus,

$$
\mathbb{I}=0, \quad \mathbb{1}=1, \quad-\mathbb{1}=2, \quad i=4 \text { or } 8 .
$$

All except $1 / 1$. The elass of all rational numbers. $a \oplus b=a+b$. $a \odot b=a b$ when $a b=1$; otherwise $a \odot b$ not in the class.

All except M2. The class of eomplex numbers of the form $a e_{1}+b e_{2}$, where $a$ and $b$ are any rational numbers, and the "units," $e_{1}$ and $e_{2}$, are eonnected by the following "multiplieation table :"*

$$
e_{1} e_{1}=e_{1} ; e_{1} e_{2}=e_{2} e_{1}=-e_{2} ; e_{2} e_{2}=-e_{1} \text {. }
$$

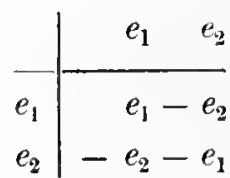

All except $M 3_{1}$ and $M 5$. The elass of all complex numbers of the form $a e_{1}+b e_{2}$ where $a$ and $b$ are positive rational numbers or zero, and *

$$
e_{1} e_{1}=e_{1} ; e_{1} e_{2}=e_{1} ; e_{2} e_{1}=e_{2} ; e_{2} e_{2}=e_{2}
$$

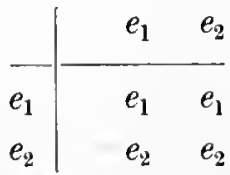

All except $M 3_{2}$ and $M 5$. The same class as the preeeding, with

$$
e_{1} e_{1}=e_{1} ; e_{1} e_{2}=e_{2} ; e_{2} e_{1}=e_{1} ; e_{2} e_{2}=e_{2}
$$

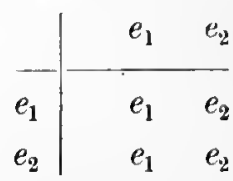

\begin{tabular}{|c|c|}
\hline & $e_{1}$ \\
\hline$e_{1}$ & $e_{1}$ \\
\hline$e_{2}$ & $e_{2}$ \\
\hline
\end{tabular}

All except $M 3_{1}$ and $1 / 3_{2}$. The elass of all eomplex numbers of the form $a e_{1}+b e_{2}$, where $a$ and $b$ are any rational numbers and *

$$
e_{1} e_{1}=e_{1} ; e_{1} e_{2}=e_{2} e_{1}=e_{2} ; e_{2} e_{2}=e_{2}
$$

*It is understood that the "sum" of two complex numbers $a e_{1}+b e_{2}$ and $a^{\prime} e_{1}+b^{\prime} e_{2}$ is $\left(a+a^{\prime}\right) e_{1}+\left(b+b^{\prime}\right) r_{2}$ their "product" is $a a^{\prime} e_{1} e_{1}+a b^{\prime} e_{1} e_{2}+b c^{\prime} e_{2} e_{1}+b b^{\prime} e_{2} e_{2}$, where the expressions $e_{1} e_{1}, e_{1} e_{2}, e_{2} \rho_{1}$ and $e_{2} \rho_{2}$ are to be simplified, in any given case, according to the "multiplication-table" adopted in that case. Iu any such system of complcx numbers, botl the distributive laws (M 4 ) will clearly be satisfled; moreover, the associative and commutative laws for multiplication $(M 2, M 5)$ will hold throughout the system whenever they hold for the "multiplication-table" of the $e$ 's. 
All except $M 4_{1}$ and $M 5$. The class of all couples of the form $(a, b)$, where $a$ and $b$ are positive rational numbers, with $\oplus$ and $\odot$ defined as follows :

$$
\begin{aligned}
& \left(a_{1}, b_{1}\right) \oplus\left(a_{2}, b_{2}\right)=\left(a_{1}+a_{2}, b_{1}+b_{2}\right) ; \\
& \left(a_{1}, b_{1}\right) \odot\left(a_{2}, b_{2}\right)=\left(a_{1} a_{2}, a_{1} b_{2}+b_{1}\right) .
\end{aligned}
$$

All except $M 4_{2}$ and $M 5$. The same elass as the preeding, with $\oplus$ defined in the same way, and $\odot$ defined as follows:

$$
\left(a_{1}, b_{1}\right) \odot\left(a_{2}, b_{2}\right)=\left(a_{1} a_{2}, b_{1} a_{2}+b_{2}\right) .
$$

All except $M 4_{1}$ and $M / 4_{2}$. The elass of all rational numbers.

$$
a \oplus b=a+b . \quad a \odot b=a+b+1 .
$$

All except M5. The class of all eomplex numbers of the form $a e_{1}+b e_{2}$ $+c e_{3}+d e_{4}$, with the following "multiplieation table" for the "units" $e_{1}, e_{2}, e_{3}$, and $e_{4}$ :

\begin{tabular}{r|rrrr} 
& $e_{1}$ & $e_{2}$ & $e_{3}$ & $e_{4}$ \\
\hline$e_{1}$ & $e_{1}$ & $e_{2}$ & $e_{3}$ & $e_{4}$ \\
$e_{2}$ & $e_{2}$ & $-e_{1}$ & $e_{4}$ & $-e_{3}$ \\
$e_{3}$ & $e_{3}$ & $-e_{4}$ & $-e_{1}$ & $e_{2}$ \\
$e_{4}$ & $e_{4}$ & $e_{3}$ & $-e_{2}$ & $-e_{1}$
\end{tabular}

This is the system of quaternions with rational coefficients.

Thus the independenee of all the postulates of $\$ 1$ (except A5) is established. The redundancy of postulate $A 5$ is proved on page 25 .

\section{§t. PROOFS OF THEOREMS IN" $\$ \S 2-4$.* $^{*}$}

The proofs of a number of theorems in $\S \S 2-4$, were postponed to the present section, to avoid interruption in reading.

Page 9.

Proof of theorem 1. Suppose $z+z=z$ and $z^{\prime}+z^{\prime}=z^{\prime}$. Then $z+z+z^{\prime}=z+z^{\prime}+z^{\prime}$, by $A 1,2$; henee $z+z^{\prime}=z^{\prime}+z^{\prime}$, by $A 3_{1}$, and theretore $z=z^{\prime}$, by $A 3_{2}$.

* I am indebted to Mr. P. W. Bridgman and Mr. G. C. Evans for assistance in verifying the demonstrations in this and the preceding section. 


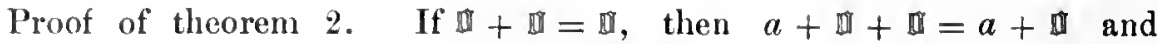
$\mathbb{I I}+\mathbb{I}+a=\mathbb{I}+a$, by $A 1,2$; henee $a+\mathbb{I}=a$, by $A 3_{2}$, and $\mathbb{I}+a=a$, by $A 3_{1}$.

Conversely, if $a+x=a$ or $x+a=u$, then $a+x+x=a+x$ or $x+x+a=x+a$, by $A 1,2$; henee, $x+x=x$, by $A 3$.

Proof of theorem 3. By M1, $4_{1}, a \times \mathbb{a}=a \times(\mathbb{I}+\mathbb{I})=(a \times \mathbb{\mathbb { a }})$ $+(a \times \mathbb{4})$, whence $a \times \mathbb{U}=\mathbb{I}$, by theorem 1 ; again, by $M 1,4_{2}, \mathbb{I} \times a$ $=(\mathbb{1}+\mathbb{I}) \times a=(\mathbb{a} \times a)+(\mathbb{a} \times a)$, whenee $\mathbb{I} \times a=\mathbb{a}$, by theorem 1 .

Proof of theorem 4. If $a \times b=\mathbb{I}$, then $a \times b=a \times \mathbb{1}$, by theorem 3 ; hence, if $a \neq \mathbb{M}$, then $b=\mathbb{I}$, by $M 3_{\mathrm{I}}$. Or thus: if $a \times b=\mathbb{\mathbb { H }}$, then $a \times b$ $=\mathbb{} \times b$, by theorem 3 ; hence, if $b \neq \mathbb{a}$, then $a=\mathbb{I}$, by $M 3_{2}$.

l'roof of theorem 5 . Suppose $u u=u$ and $u^{\prime} u^{\prime}=u^{\prime}$, with $u \neq \mathbb{\|}$ and $u^{\prime} \neq \mathbb{1}$. Then $u u u^{\prime}=u u^{\prime} u^{\prime}$, by $M / 1,2$; henee $u u^{\prime}=u^{\prime} u^{\prime}$, by $M 3_{1}$, and therefore $u=u^{\prime}$, by $1 / 3_{2}$.

Proof of theorem 6 . If $\mathbb{1} \times \mathbb{1}=\mathbb{1}$, then $a \times \mathbb{1} \times \mathbb{1}=a \times \mathbb{1}$ and $\mathfrak{l} \times \mathfrak{l} \times a=\mathbb{1} \times a$, by $\mathbb{N} 1,2$; henee $a \times \mathbb{1}=a$, by $M / 3_{2}$, and $\mathbb{l} \times a=a$, by $U 3_{1}$, sinee $\mathfrak{l} \neq \mathbb{\pi}$.

Pages 11-12.

Proof of theorem 7. If $a x=a$ or $x a=a$, then $a x x=a x$ or $x x a=x a$, by $M / 1,2$; henee $x x=x$, by $M 3$, so that $x=\mathbb{I}$ or $\mathbb{1}$. But $x \neq \mathbb{a}$, sinee if $x=\mathbb{I}$, then $a=\mathbb{I}$, by theorem 3 ; therefore $x=\mathbb{l}$.

Proof of lemma to theorem 8. If $a+b=\mathbb{I}$, then $b+a+b=b+\mathbb{U}$ $=b=\mathbb{I}+b$, by $A 1,2$, and theorem 2 ; henee $b+a=\mathbb{a}$, by $A 3$. . Theorem 8 follows from this lemma by $A B$.

Proof of theorem 9. By $M 4_{1}$ and theorem $3, a(-b)+a b=a(-b+b)$ $=a \times \mathbb{U}=\mathbb{a}$, so that $a(-b)$ and $a b$ are opposite elements; again, by $M 4_{\mathrm{e}}$ and theorem $3,(-a) b+a b=(-a+a) b=\mathbb{1} \times b=\mathbb{1}$, so that $(-a) b$ and $a b$ are opposite elements. Henee, further, $(-a) \times(-b)=-[(-a) \times b]$ $=-[-(a b)]=a b$, by the eorollary to theorem 8 .

Proof of theorem 10. By A2, 5, $(-a)+(-b)+(a+b)=(-a)$ $+a+(-b)+b=\mathbb{a}+\mathbb{d}=\mathbb{f}$, so that $(-a)+(-b)$ and $(a+b)$ are opposite elements.

Proof of theorem 12. First, $[a+(b-c)]+c=a+[(b-c)+c]$ $=a+b=[(a+b)-c]+c$; hence $a+(b-c)=(a+b)-c$, by $A 3_{2}$. The seeond equation is proved in a similar way by adding $b+c$ to both sides, and the third equation by adding $b$. 
Proof of theorem 13. By $M 4_{1}, a c+a(b-c)=a[c+(b-c)]$ $=a b=a c+(a b-a c)$; henee $a(b-c)=a b-a c$, by $M 3_{1}$. Similarly for the seeond part of the theorem.

Proof of lemma to theorem 14. If $a \neq \mathbb{1}$ and $a b=\mathfrak{d}$, then $a b a=\mathbb{l} \times a$ $=a \times \mathbb{1}$, whenee $b a=\mathbb{d}$, by $M 3_{1}$. If $b \neq \mathbb{\mathbb { N }}$ and $a b=\mathbb{1}$, then $b a b=b \times \mathbb{1}$ $=\mathbb{1} \times b$, whenee $b a=\mathbb{1}$, by $M 3_{2}$. If both $a$ and $b$ are $\mathbb{U}$, then in any ease $a b=b a$.

Proof of theorem 14. If $a y=\mathbb{1}$ and $a y^{\prime}=\mathbb{1}$, then, by the lemma, $y^{\prime}=\mathfrak{l} \times y^{\prime}=y a \times y^{\prime}=y \times a y^{\prime}=y \times \mathbb{1}=y$.

Pages 13-14.

Proof of theorem 15. $a\left(\frac{\mathbb{d}}{a}\right)=\mathbb{1}=(-a)\left(\frac{\mathbb{1}}{-a}\right)=a\left[-\left(\frac{\mathbb{1}}{-a}\right)\right]$, by theorem 9 ; henee $\frac{\mathfrak{l}}{a}=-\left(\frac{\mathfrak{d}}{-a}\right)$, by $M 3_{1}$.

Proof of theorem 16. $\quad \frac{\mathbb{1}}{a} \times \frac{\mathbb{1}}{b} \times a b=\frac{\mathfrak{l}}{a} \times a \times \frac{\mathbb{1}}{b} \times b=\mathbb{1} \times \mathbb{1}=\mathbb{l}$, so that $\frac{\mathbb{l}}{a} \times \frac{\mathbb{1}}{b}$ and $u b$ are reciproeal elements.

Proof of theorem 18. Multiply the five equations by $b c, b, b c, b d, c / d$, respeetively; then use $M B$.

Proof of theorem 19. Multiply the equations by $b l^{\prime}$, and reduee by $1 / 4$; then use $M 3$, sinee $b$, and $b^{\prime}$ must be different from $\mathbb{~ I I}$.

Proof of theorem 20. First, in any system containing the elements $\&$ and $-\mathfrak{l}$, every element will have an opposite, sinee, if $x$ is any element, then $(-\mathbb{1}) \times x$ will exist in the system, by,$V 1$, and $(-\mathbb{1}) \times x=-x$, by theorems 9 and 6 ; moreover, if $x x=-\mathbb{1}$, then $(-x) \times(-x)=-1$, by theorem 9. Seeondly suppose $x x=-\mathbb{l}$ and $y y=-\mathfrak{l}$; then $x x-y y=\mathbb{l}$, whenee $(x+y)(x-y)=\mathbb{I}$, by $M 4,5$, and therefore either $x+y=\mathbb{I}$ or $x-y=\mathbb{I}$, by theorem 4 ; that is, $x=-y$ or $x=y$.

Page 16.

Proof of the laws of addition and multiplication for the positive integers.

$$
\underline{(\alpha+\beta)+\gamma=a+(\beta+\gamma)} .
$$

This is true when $\gamma=1$, by definition. Also, if it is true when $\gamma=\nu$, then it will be true when $\gamma=\nu^{\prime}$, where $\nu^{\prime}$ is the sueessor of $\nu$; for, $(a+\beta)+\nu^{\prime}=[(a+\beta)+\nu]+1=[a+(\beta+\nu)]+1=a+[(\beta+\nu)+1]=a+\left(\beta+\nu^{\prime}\right)$. 
Hence, putting successively $\nu=1,2,3, \ldots$, we see that the formula is true when $\gamma$ is any positive integer. This method of proof is called induction from $\gamma=\nu$ to $\gamma=\nu^{\prime}$, when $\nu^{\prime}$ is the successor of $\nu$; or brietly, induction on $\gamma$.

2) Lemma. $a+1=1+a$. By induction on $\boldsymbol{a}$ : $\nu^{\prime}+1=(\nu+1)+1=(1+\nu)+1=1+(\nu+1)=1+\nu^{\prime}$.

3) $\quad a+\beta=\beta+a$.

For, by induction on $\beta$ : $a+\nu^{\prime}=(a+\nu)+1=(\nu+a)+1=\nu+(a+1)=\nu+(1+a)=(\nu+1)+a=\nu^{\prime}+a$.

4) $a(\beta+\gamma)=a \beta+a \gamma$. For, by induction on $a$ : $\nu^{\prime}(\beta+\gamma)=\nu(\beta+\gamma)+(\beta+\gamma)=\nu \beta+\nu \gamma+\beta+\gamma=\nu \beta+\beta+\nu \gamma+\gamma=\nu^{\prime} \beta+\nu^{\prime} \gamma$.

5) $(\beta+\gamma) a=\beta a+\gamma a$.

For, by induction on $\gamma$ : $\left(\beta+\nu^{\prime}\right) a=[(\beta+\nu)+1] a=(\beta+\nu) a+a=\beta a+\nu a+a=\beta a+\nu^{\prime} \alpha$.

6) $(\alpha \beta) \gamma=a(\beta \gamma)$.

For, by induction on $a$ : $\left(\nu^{\prime} \beta\right) \gamma=(\nu \beta+\beta) \gamma=(\nu \beta) \gamma+\beta \gamma=\nu(\beta \gamma)+\beta \gamma=\nu^{\prime}(\beta \gamma)$.

Lemma. $\quad 1 a=a \cdot 1$

For, by induction on $a$ :

$$
1 \nu^{\prime}=1(\nu+1)=1 \nu+1 \cdot 1=\nu \cdot 1+1=\nu^{\prime} \cdot 1 .
$$

$$
\begin{array}{ll}
\frac{a \beta=\beta a .}{\nu^{\prime} \beta=\nu \beta+\beta=\beta \cdot \nu+\beta \cdot 1=\beta(\nu+1)}=\beta \nu^{\prime} .
\end{array}
$$

For, by induction on $a$ :

9) Lemma. If $a \neq \beta$, then either $a=\beta+\xi$ or $\beta=a+\xi$, where $\xi$ is some positive integer. For, if $a>\beta$, then $a$ will be found in the sequence $\beta+1, \beta+2, \beta+3, \ldots$; and if $\beta>a$, then $\beta$ will be found in the sequence $a+1, a+2, a+3, \cdots$

10) Lemma. $a+\beta \neq a$. For, $a+\beta$ will be found in the sequence $a+1, a+2, a+3, \cdots$, and each of these numbers is different from $a$.

11) If $a+\xi=a+\eta$, then $\xi=\eta$. For, if $\xi \neq \eta$, then we should have $\xi=\eta+\zeta$, say, whence $a+(\eta+\zeta)=a+\eta$, or $(a+\eta)+\zeta=(a+\eta)$, which is impossible.

12) If $a \xi=a \eta$, then $\xi=\eta$. For, if $\xi \neq \eta$, then we should have $\xi=\eta+\zeta$, say, whenee $a(\eta+\zeta)=a \eta$, or $a \eta+a \zeta=a \eta$, which is impossible.

Proof of theorem 22. By definition, $\lambda a+a=(\lambda+1) a$; hence $\lambda a+\mu \iota$ $=(\lambda+\mu) a$, by induction on $\mu$, since $\lambda u+\nu^{\prime} a=\lambda a+(\nu a+a)=(\lambda a+\nu a)+a$ $(\lambda+\nu) a+a=[(\lambda+\nu)+1] u=\left(\lambda+\nu^{\prime}\right) u$. 
Again, $1(\mu a)=(1 \mu) a$; henee $\lambda(\mu a)=(\lambda \mu) a$, by induction on $\lambda$, sinee

$$
\nu^{\prime}(\mu \iota)=\nu(\mu a)+\mu a=(\nu \mu) a+\mu \iota=(\nu \mu+\mu) a=\left(\nu^{\prime} \mu\right) a .
$$

Pages 17-19.

Proof of theorem 23.

$$
\begin{gathered}
(\lambda a-\mu c)+\mu \iota=\lambda u=[(\lambda-\mu)+\mu] a=(\lambda-\mu) a+\mu a ; \text { henee } \\
\lambda a-\mu \ell=(\lambda-\mu) a, \text { by } A 3_{2 .} .
\end{gathered}
$$

Proof of theorem 24. By definition, $1(-a)+1 a=\mathbb{I I} ;$ henee $\mu(-a)$ $+\mu a=\mathbb{I}$, by induetion on $\mu$, since

$$
\nu^{\prime}(-a)+\dot{\nu}^{\prime} \iota=(-u)+[\nu(-a)+\nu(u]+u=(-a)+\mathbb{l}+a=\mathbb{a} ;
$$

therefore $\mu(-a)$ and $\mu a$ are opposite elements.

Proof of theorem 26. If there is a zero-element, $\mathbb{D}$, then every multiple

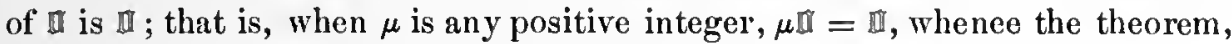
by $A 4$.

Proof of the corollary. Suppose $\lambda a=\mu a$, and $\lambda>\mu$; then $\lambda=\mu+\xi$, so that $(\mu+\xi) a=\mu a$, whenee $\mu a+\xi a=\mu a$; therefore $\xi a=\mathbb{l}$, by theorem 2 , and henee $\iota=\mathbb{I}$, by theorem 26 .

Proof of theorem 28. $\mu\left[\lambda\left(\frac{\ell}{\mu}\right)\right]=\lambda\left[\mu\left(\frac{\ell}{\mu}\right)\right]=\lambda \iota=\mu\left[\frac{\lambda \epsilon}{\mu}\right]$; henee $\lambda\left(\frac{a}{\mu}\right)=\frac{\lambda \epsilon}{\mu}$, by $A 4$.

Proof of theorem 29. Put $\xi=\frac{\lambda}{\mu}, \eta=\frac{\lambda_{1}}{\mu_{1}}$; take the $\left(\mu \mu_{1}\right)^{\text {th }}$ multiple of both sides of the equation, and use theorem 27.

Page 21.

Proof of theorems 33-35. Let $\lambda, \mu$ be any positive integers. We prove first that $a(\mu b)=\mu(a b)$; thus, $a(1 \cdot b)=1 \cdot(a b)$, and by induetion,

$$
a\left(\nu^{\prime} l\right)=a(\nu l+b)=a(\nu b)+a b=\nu(a l)+a b=\nu^{\prime}(a b),
$$

where $\nu^{\prime}$ is the sueeessor of $\nu$. Then it follows that $(\lambda(c)(\mu b)=(\lambda \mu)(a b)$; for, $(1 \cdot a)(\mu b)=(1 \cdot \mu)(a b)$; and by induetion,

$$
\begin{aligned}
\left(\nu^{\prime} a\right)(\mu b)=(\nu a+a)(\mu b)=(\nu a)(\mu b)+a(\mu b) & =(\nu \mu)(a b)+\mu(a b) \\
& =(\nu \mu+\mu) a b=\left(\nu^{\prime} \mu\right)(a b) .
\end{aligned}
$$


To prove that $\left(\frac{\lambda}{\mu} a\right)\left(\frac{\lambda_{1}}{\mu_{1}} b\right)\left(\frac{\lambda}{\mu}=\frac{\lambda_{1}}{\mu_{1}}\right)(a b)$, take the $\left(\mu \mu_{1}\right)^{\text {th }}$ multiple of both sides. The proof for negative eoeffieients follows from theorem 24 and theorem 9.

Proof of theorem 36 . Let $\lambda, \mu$ be any positive integers.

1) If $\lambda x=\lambda y$, then $x=y$, by $A 4$.

If $\frac{\lambda}{\mu} x=\frac{\lambda}{\mu} y$, then $\lambda x=\left(\mu \frac{\lambda}{\mu}\right) x=\mu\left(\frac{\lambda}{\mu} x\right)=\mu\left(\frac{\lambda}{\mu} y\right)=\left(\mu \frac{\lambda}{\mu}\right) y=\lambda y$, and therefore $x=y$ as above.

$$
\text { If }\left(-\frac{\lambda}{\mu}\right) x=\left(-\frac{\lambda}{\mu}\right) y \text {, then }-\left(\frac{\lambda}{\mu} x\right)=-\left(\frac{\lambda}{\mu} y\right) \text {, whenee } \frac{\lambda}{\mu} x=\frac{\lambda}{\mu} y \text {, and }
$$
therefore $x=y$ as above.

2) If $\lambda \iota=\mu r$, then $\lambda=\mu$, by theorem 26 .

If $\frac{\lambda}{\mu} a=\frac{\lambda_{1}}{\mu_{1}} a$, then $\mu_{1} \lambda a=\mu_{1} \mu\left(\frac{\lambda}{\mu} a\right)=\mu \mu_{1}\left(\frac{\lambda_{1}}{\mu_{1}} a\right)=\mu \lambda_{1} a$, and therefore $\mu_{1} \lambda=\mu \lambda_{1}$ as above; that is, $\frac{\lambda}{\mu}=\frac{\lambda_{1}}{\mu_{1}}$.

If $\left(-\frac{\lambda}{\mu}\right) "=\left(-\frac{\lambda_{1}}{\mu_{1}}\right) "$, then $\frac{\lambda}{\mu} "=\frac{\lambda_{1}}{\mu_{1}} "$, and therefore $\frac{\lambda}{\mu}=\frac{\lambda_{1}}{\mu_{1}}$ as above; hence $-\frac{\lambda}{\mu}=-\frac{\lambda_{1}}{\mu_{1}}$.

The proof of theorem 37 follows at onee from theorem 36 .

Proof of theorem 38. Let $\lambda, \mu$ be any positive integers.

Clearly, $1(a+b)=1 a+1 b$; henee $\lambda(a+b)=\lambda a+\lambda b$ by induetion, sinee $\nu^{\prime}(a+b)=\nu(a+b)+(a+b)=\nu a+\nu b+a+b=\nu a+a+\nu b+b$ $=\nu^{\prime} \iota+\nu^{\prime} b$, where $\nu^{\prime}$ is the successor of $\nu$.

Further, $\frac{\lambda}{\mu}(a+b)=\frac{\lambda}{\mu} a+\frac{\lambda}{\mu} b$, since the $\mu^{\text {th }}$ multiple of each side is $\lambda(a+b)$ or $\lambda a+\lambda b$.

Finally, $\left(-\frac{\lambda}{\mu}\right)(a+b)=\left(-\frac{\lambda}{\mu}\right) a+\left(-\frac{\lambda}{\mu}\right) b$, by the aid of theorem 10.

Proof of theorem 39. $\xi(a-b)+\xi b=\xi(a-b+b)=\xi a=(\xi a-\xi b)$ $+\xi b$; hence $\xi(a-b)=\xi a-\xi b$, by $A 3_{2}$. 
Proof of theorem 40.

$(\eta b)\left(\frac{\xi a}{\eta b}\right)=\xi \iota=\left(\eta \frac{\xi}{\eta}\right)\left(b_{\bar{b}}^{a}\right)=(\eta b)\left(\frac{\xi}{\eta} \cdot \frac{a}{b}\right)$; hence $\frac{\xi_{\iota}}{\eta b}=\frac{\xi}{\eta} \cdot \frac{\imath}{b}$, by $1 / 3_{1}$, since $\eta \bar{b} \neq \mathbb{\mathbb { I }}$.

The proofs of theorems $41-45$ are similar to the proofs of theorems 2125 , and need not be given here in detail.

Page 24.

Proof of lemma to theorem 50. If $x=a$ is a root of the equation

then

$$
c_{0} x^{\mu}+c_{1} x^{\mu-1}+c_{2} x^{\mu-2}+\cdots+c_{\mu-1} x+c_{\mu} \cdot \mathbb{1}=\mathbb{I} \text {, }
$$

$$
c_{0} \tau^{\mu}+c_{1} \tau^{\mu-1}+c_{2} \tau^{\mu-2}+\cdots+c_{\mu-1} t^{\ell}+c_{\mu} \cdot \mathbb{l}=\mathbb{I} ;
$$

henee, by subtraction, the given equation may be written in the form $c_{0}\left(x^{\mu}-\iota^{\mu}\right)+c_{1}\left(x^{\mu-1}-\iota^{\mu-1}\right)+c_{2}\left(x^{\mu-2}-\iota^{\mu-2}\right)+\cdots+c_{\mu-1}(x-\iota)=\mathbb{I}$, eaeh term of whieh, by theorem 49 , is divisible by $x-a$.

\section{APPENDIX 1.}

The following postulate holds in all the types of algebra which we have eonsidered in this paper:

Postulate E7. If $x \neq y$, then there is either an element $v$ such that $x=y+v$, or an element $w$ such thut $y=x+w$.

In the first case, $v=a-b$; in the second, $w=b-a$ (compare theorem 11).

If we add this postulate $E 7$ to the list of general laws in $\S 1$, then postulates $A 4$ and $M 3$ may be replaced by the following simpler postulates, $A 4^{\prime}$ and $M 3^{\prime}$ :

Postulate $A 4^{\prime}$. If $a \neq \mathbb{\square}$, then $\mu a \neq \mathbb{\square}$, where $\mu$ is cony multiple of $a$ (see theorem 21).

This is a modified form of the law of uon-circularity.

Postulate $M 3^{\prime}$. If $a \neq \mathbb{I}$ and $b \neq \mathbb{d}$, then $a b \neq \mathbb{I}$.

This is the law of the zero-produet (compare thcorem 4).

The deduction of postulate $A 4$ from $A 1,2,3,5$ and $E 7$ is as follows:

We are to prove that if $\mu x=\mu y$, then $x=y$. Suppose $x \neq y$, and that 
$x=y+v$, by $E 7$. Then $\mu x=\mu y+\mu v$, by theorem 38 for positive integers; whence $\mu z^{\prime}=\mathbb{B}$, by hypothesis and theorem 2, so that $v=\mathbb{Z}$, by $A 4^{\prime}$ '. 'Therefore $x=y$, by theorem 2. - Similarly if $y=x+w$. follows:

The deduetion of postulate $M 3_{1}$ from $A 1,2,3, M 1,3^{\prime}, 4_{1}$, and $E 7$ is as

We are to prove that if $a x=a y$ and $a \neq \mathbb{\square}$, then $x=y$. Suppose $x \neq y$, and that $x=y+v$, by $E 7$. Then $a x=a y+a v$, by $M 4_{1}$. whence $a v=\mathbb{I}$, by lyypothesis and theorem 2, so that $v=\mathbb{J}$, by $M 3^{\prime}$ (since $a \neq \mathbb{I}$ ). Therefore $x=y$, by theorem 2. - Similarly if $y=x+w$. and $E 7$.

The deduction of $M / 3_{2}$ follows in like manner from $A 1,2,3, M 1,3^{\prime}, 4_{2}$,

\section{Examples.}

An example of a system whiel satisfies $A 1-A 5$ and $M 1-M 5$, but not $E 7$, is the system of all positive rational numbers $>2$, with addition and multiplieation defined in the usual way.

An example of a system whiel satisfies $A 1,2,3,4,5$ and $M 1,2,3 \prime, 4,5$, but not $1 / 3$, is the system of all complex numbers of the form $a e^{\prime}+b e^{\prime \prime}$, or $(a, b)$, where $a$ is zero or any positive rational number, and $b$ is zero or a positive rational $>1$, with the following "multiplieation table" for the units :

$$
e^{\prime} e^{\prime}=e^{\prime} ; e^{\prime} e^{\prime \prime}=e^{\prime \prime} e^{\prime}=e^{\prime \prime} ; e^{\prime \prime} e^{\prime \prime}=e^{\prime \prime}
$$

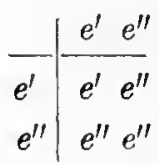

This system eontains a zero-element, $\mathbb{\square}=(0,0)$, and a unit-element, $\mathbb{1}=(1,0)$. To show that it does not satisfy $M 3$, note that $(0,2) \odot(4,5)$ $=(0,18)$ while also $(0,2) \odot(3,6)=(0,18)$. Moreover, as was to be expected, it does not satisfy $E 7$; for example, if $a=(2,7)$ and $b=(3,6)$, then neither $a-b$ nor $b-a$ exists in the system.

The existenee of this system shows that the set of postulates in $\$ 1$ is "weakened"* when $M 3$ is replaced by $M 3^{\prime}$, sinee $M / 3$ eannot be deduced from $\triangle / 3^{\prime}$ without the aid of an additional postulate, like $E 7$.

An example of a system satisfying $A 1,2,3,4^{\prime}, 5$ and $M 1,2,3,4,5$, but not $A 4$, would also be interesting; I have not, however, been able to find an

* The notion "weaker" seems to me to be applicable rather to a set of postulates than to a single postulate. 
example of this kind. It therefore remains an open question whether the set of postulates in $\$ 1$ is really "weakened" when $A 4$ is replaeed by $A 4$ '.

\section{APPENDIX 2.}

It may be interesting to note here, somewhat more in detail than in the text, what can be done with postulates $A 1,2,3 ; M 1,2,3$ without the aid of the distributive laws for multiplieation.

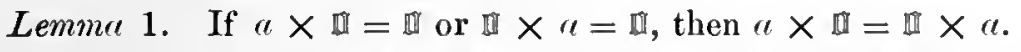

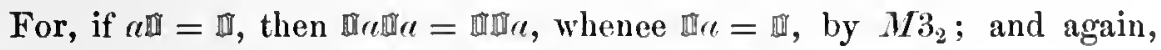

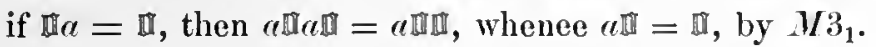

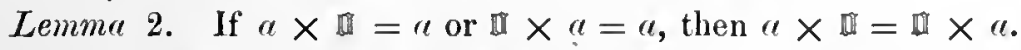

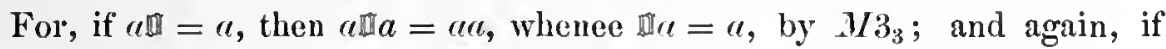

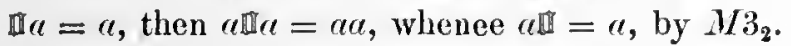

Theorem $A$. In any system whieh satisfies $A 1,2,3 ; M 1,2,3$, if we assume in regard to the multiplieative property of $\mathbb{I}$, merely that

$$
\text { 田 } \times \mathbb{I}=\mathbb{I},
$$

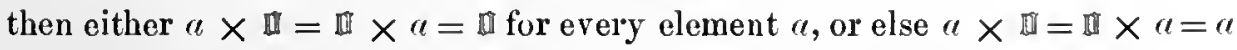
for every element $\boldsymbol{~}$.

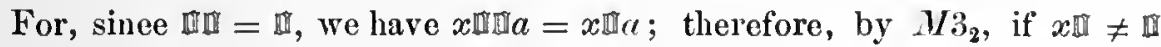
for any single element $x$, then $\mathbb{I} \iota=a$ for every element $a$. Hence the theorem, by lemmas 1 and 2 .

Theorem $B$. In any system whieh satisfies $A 1,2,3 ; M 1,2,3$, if

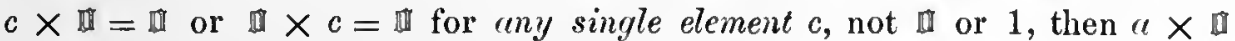
$=\mathbb{I I} \times \alpha=\mathbb{I}$ for every element $\alpha$.

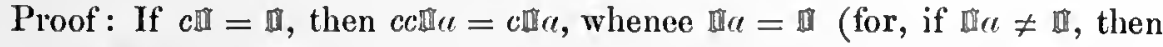
$c c=c$, by $M 3_{2}$, and therefore $c=\mathbb{U}$ or $\mathfrak{l}$, by theorem 8 ); henee the theorem, by lemma 1 .

Theorem $C$. In any system whieh satisfies $A 1,2,3 ; M 1,2,3$, if

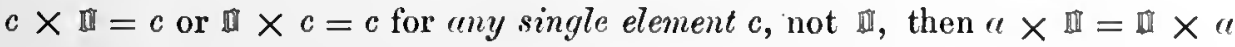
$=a$ for every element $a$.

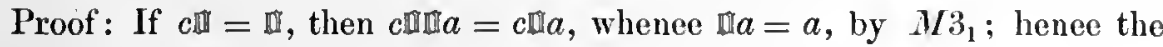
theorem, by lemma 2 . 


\section{Examples.}

The following three systems, all of which satisfy $A 1,2,3,4,5$ and $M 1$, $2,3,5$, but not $1 / 4$, will ilhstrate these theorems. In cach of the systems there is a zero-clement, namely $\mathbb{I}=0$.

Exremple 1. The class of all even integers, with addition defined in the nsual way, and multiplication defined as follows:

$$
a \odot 0=0 \odot a=a \text {; otherwise } a \odot b=a b \text {. }
$$

In this srstem, the equations $\theta \odot \mathbb{U}=\mathbb{U} \odot \theta=\mathbb{U}$ are true when and only when $\because=\mathbb{4}$. There is no unit-element.

Example 2. The class of all positive integers and zero, with $a \oplus b=$ $u+b$, and $q \odot b$ defined as follows:

$$
" \odot 1=1 \odot u=a \text {; otherwise } a \odot b=a+b+2 \text {. }
$$

In this system, there is a mit-element, namely $\mathfrak{l}=1$, and the equations

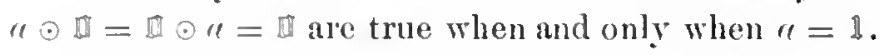

Exrmple 3. The class of all positive integers and zero, with $a \oplus b=$ $u+b$, and $a \odot b=a+b+1$.

In this system, the equations $a \odot \mathbb{I}=\mathbb{1} \odot a=\mathbb{I}$, and also the equations $a \odot \mathbb{I}=\mathbb{I} \odot n=u$, are filse for all values of $u$. There is no unit-element.

It shonld be noticed that all these systems possess the property that a product is never zero inless at least one of its factors is zero.

Halvard UNTyersitr,

Canmmide, Mass. 


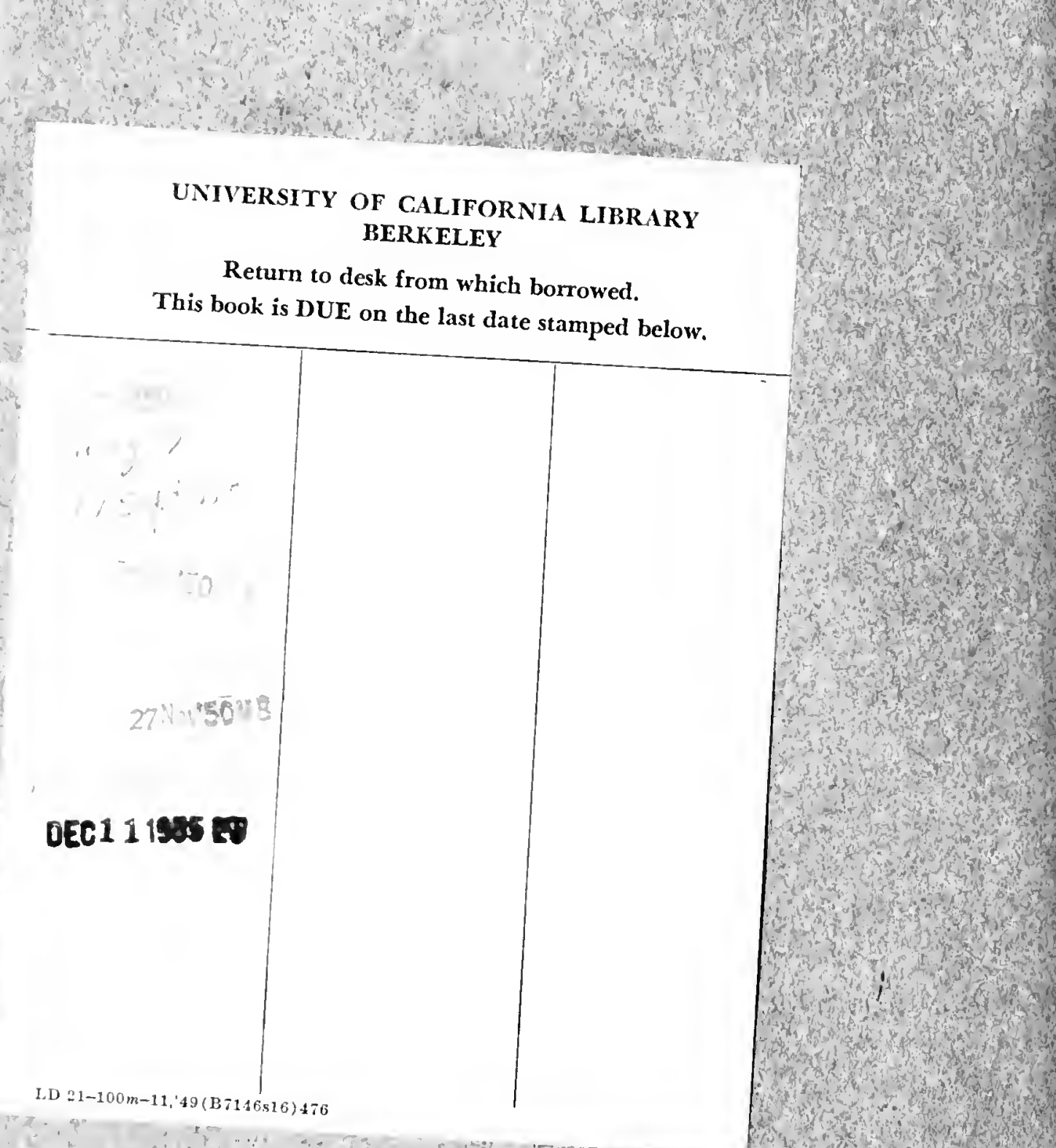

$7 x=2,2$ es
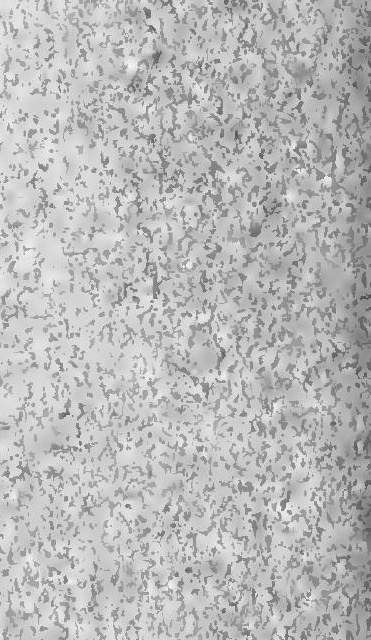

1 (12
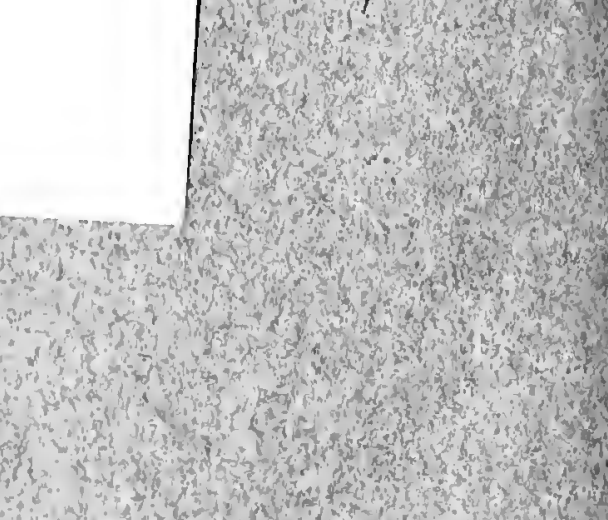
(1)

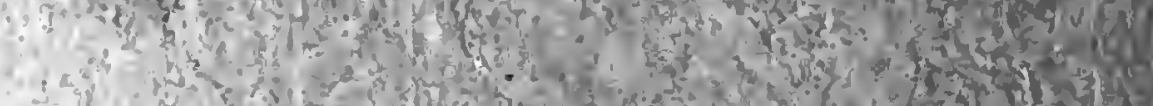

$\therefore$ is

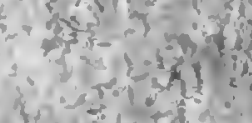

\section{and}

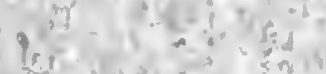

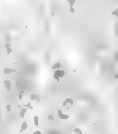

19.

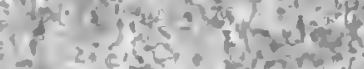

1.

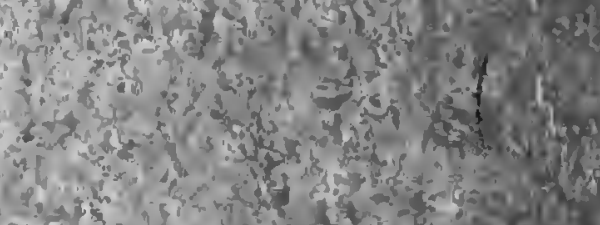

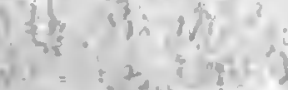

1.5

xid

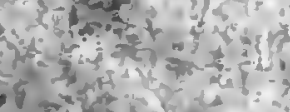

a

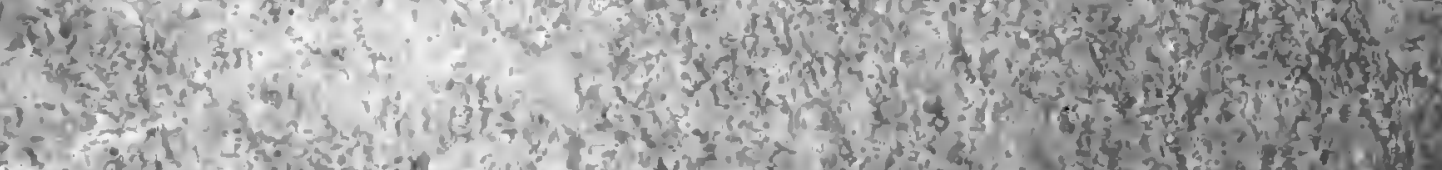

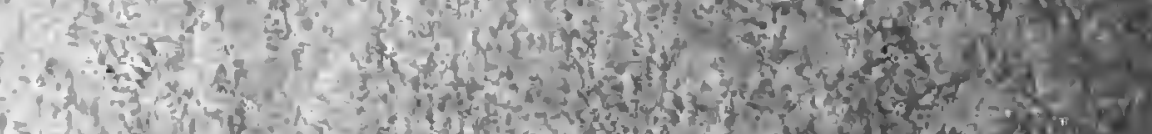

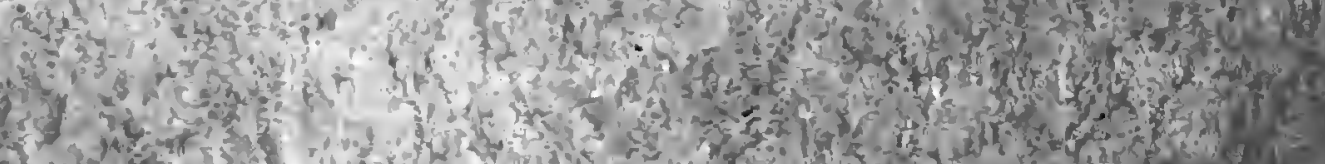

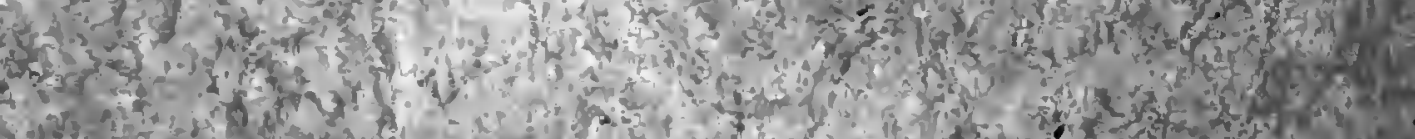

3.

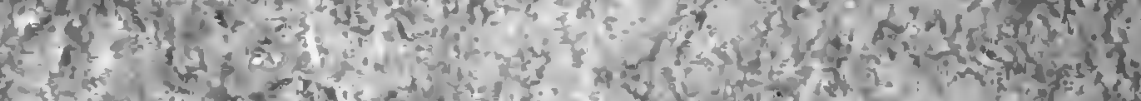

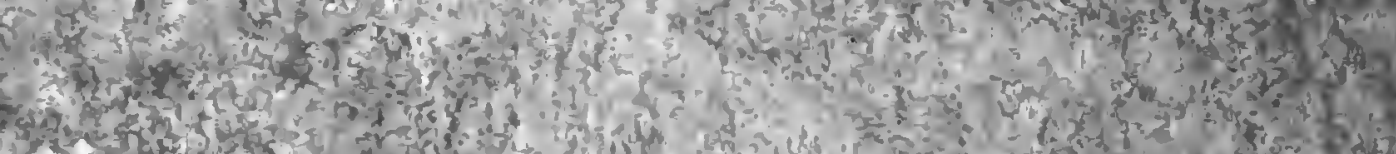

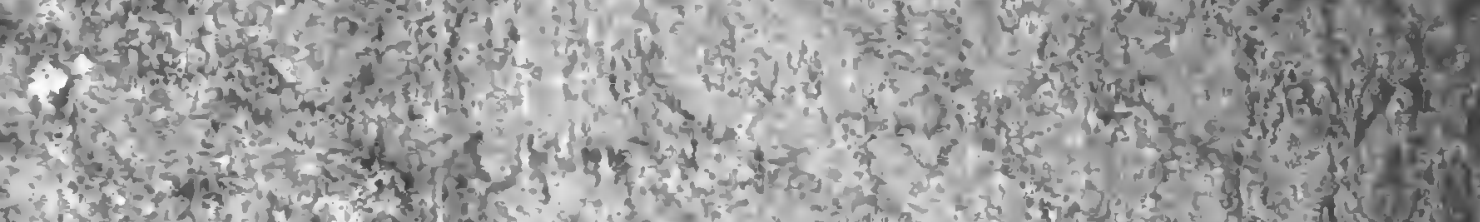
(5) S.m.

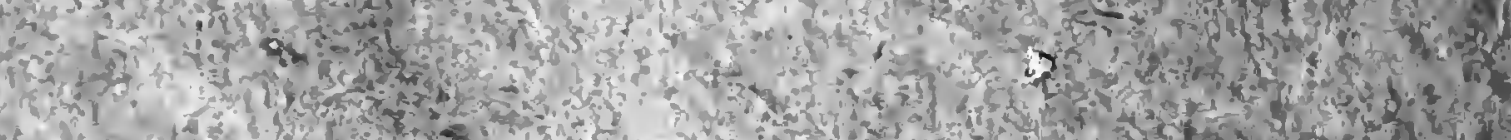

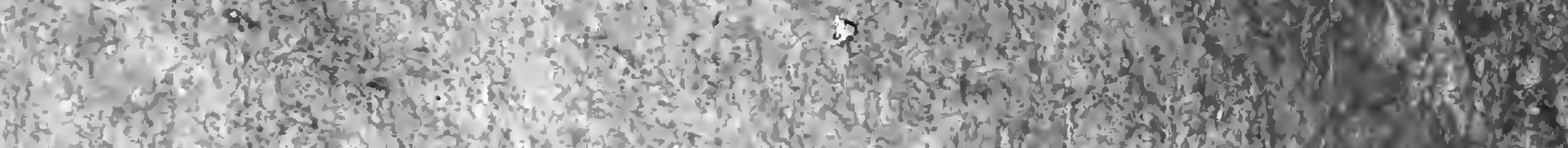
1
4

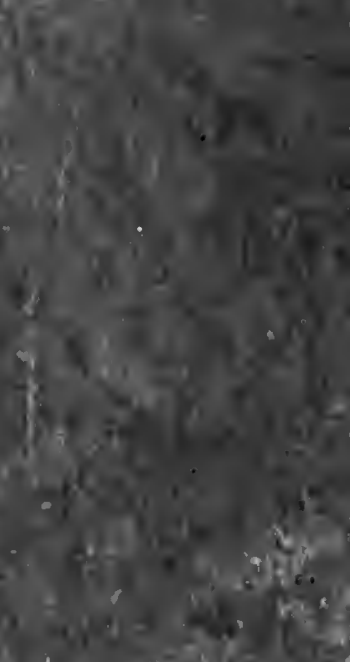

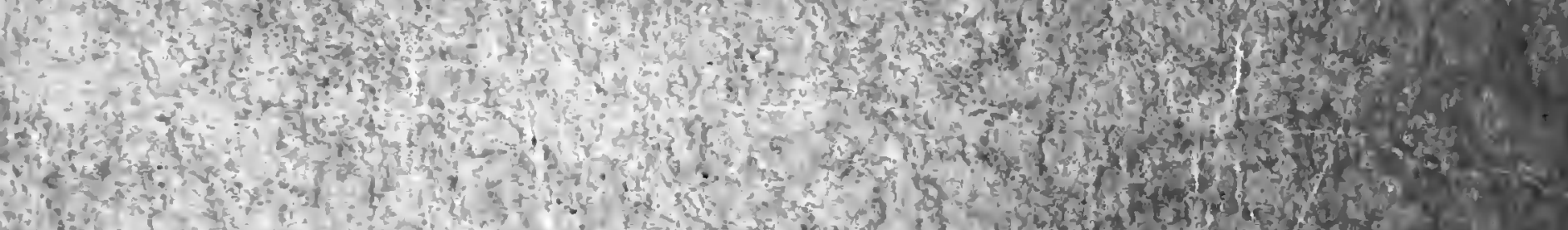

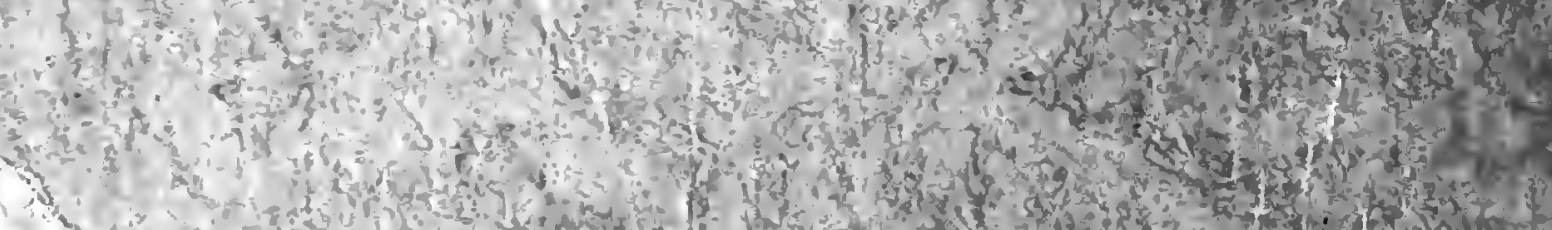

$$
\text { (i) }
$$

